\title{
EL POLEN DE HELICTERES (STERCULIACEAE) Y SU COMPARACIÓN CON GÉNEROS VECINOS
}

\author{
por STELLA M. PIRE' Y CARMEN L. CRISTÓBAL²
}

\begin{abstract}
Summary
Pollen grains of 41 species representing all seven sections of Helicteres were studied with light and scanning electron microscopy and described. In order to establish palynological affinities Neoregnellia cubensis, Kleinhovia hospita, Reevesia thyrsoidea, Veeresia clarkii, Ungeria floribunda and Pterospermum acerifolium were also examined. Helicteres is palynologically rather uniform with respect to the shape and size of the grains as well as the type and number of the apertures. The pollen grains are usually triporate, oblate or suboblate, amb triangular and medium sized. Nevertheless the exine surface shows great variability. Nine pollen types are recognized on the basis of the sculpture of the exine: Type l, tectate-perforate, baculate, in sect. Helicteres; Type II, tectate-perforate, psilate to weakly verrucate, in 4 species of sect. Orthocarpaea; Type III, tectate-perforate, with the equatorial zone verrucate and the poles psilate, in sect. Stegogamos; Type IV, microreticulate, verrucate, in sect. Polyandria; Type V, tectate, scabrate, verrucate, in sect. Alicteres; Type VI, tectate-perforate, verrucate, in 2 species of sect. Orthocarpaeaand 4 species of sect. Orthothecium; Type VII, tectate-perforatefossulate, verrucate, the verrucae large, irregular in outline, often anastomosed, in 3 species of sect. Orthothecium; Type VIII, tectate to tectate-perforate-fossulate, perforations and fossulae as well as micro-verrugae and micro-echinae densely concentrated at the poles, the equatorial zone psilate or scabrate, in 3 species of sect. Orthothecium; Type IX, tectateperforate-fossulate, microechinate, the perforations and fossulae densely concentrated at the poles, the micro-echinae distributed throughout the surface but hardly differentiated on the poles, in 16 species of sect. Sacarolha and 5 species of sect. Orthothecium. The pollen types are ordered according to the complexity of the exine; with the types with uniform sculpture considered simpler and the types with polar and equatorial zones differentiated considered complex. Keys to identify the nine pollen types of Helicteres and the pollen types of related genera are presented. According to pollen morphology Neoregnellia is closely connected with Helicteres; this genus shares the same pollen type, IX, with sect. Sacarolha and some species of sect. Orthothecium. The pollens of Kleinhovia and Helicteres have many characters in common (shape, size, apertures), the only difference being the microreticulate surface of the former; they are rather close to each other. Reevesia thyrsoidea, Veeresia clarkii and Ungeria floribunda stand apart from Helicteres in having 3-5 brevi-colp(or)ate and suprareticulate grains. The very distinctive pollen of Pterospermum acerifolium (large size, spheroidal, echinate) supports its segregation from the tribe Helictereae. In Helicteres the different patterns of exine sculpture have taxonomic and phylogenetic value. In the first place, they allow the recognition of the 4 monospecific sections (Helicteres, Stegogamos, Alicteres and Polyandria) and at the same time they reveal the coherence of the genus, since the other 3 sections which have many species (Orthocarpaea, Orthothecium and Sacarolha) are connected with each other not only by exomorphological characters but also by pollen characters. In the second
\end{abstract}

\footnotetext{
${ }^{1}$ Facultad de Ciencias Exactas y Naturales y Agrimensura, Universidad Nacional del Nordeste, 3400 Corrientes, Rep. Argentina.

${ }^{2}$ Instituto de Botánica del Nordeste (UNNE-CONICET). C.C. 209, 3400 Corrientes, Rep. Argentina.
} 
place, they provide bases for interpreting infra-generic relationships and the possible origin of the genus. Two possible evolutionary trends of exine sculpture, which are representated in two diagrams, are proposed. The first possibility starts with the Type VI; from this pollen type the evolutionary trend would have diverged in several directions. On one side, a line would lead toward increasing complexity of the exine that would end in Type VIII. This kind of pollen grain would have given rise to Type $\mathrm{X}$. On the other side, several divergent lines might have taken place; modifications in the exine scuipture would have led toward a progressive simplicity that would culminate in the absence of sculptural elements of Type II. From this kind of pollen grain Type I might have evolved. The second possibility starts from Type II; in this case the evolutionary trend of the exine would have gone in only one direction; the exine would have acquired more and more complexity, giving rise to pollen types connected with each other by transitional forms.

Key words: pollen, taxonomy, Helicteres, Helictereae

Palabras clave: polen, taxonomía, Helicteres, Helictereae

\section{Introducción y antecedentes}

Helicteres L. es un género de Sterculiaceae que no ha sido muy estudiado desde el punto de vista palinológico y los únicos antecedentes que existen sobre la morfología polínica incluyen descripciones basadas sólo en observaciones con microscopio óptico.

Uno de los primeros en referirse al polen de Helicteres fue. Winkler (1906: 255), quien describe los granos de $H$. isora como esféricos y levemente reticulados. Rao (1950) estudia el polen de las Sterculiaceae y menciona que en Helicteres los granos son oblatos y triangulares, con la exina finamente granulada y la intina engrosada alrededor de las aperturas. Erdtman (1966: 417, Fig. 239-D) describe, junto con el de otras Helictereae, los granos de $H$. angustifolia como 3 (-5)-colpor(oid)ados, brevicolpados y angulaperturados, oblatos; incluye el dibujo de una vista polar y un palinograma pero no hace mención al tipo de ornamentación. Posteriormente Salgado-Labouriau (1973) interpreta la figura de Erdtman como pilas muy esparcidas. A la misma conclusión llegan Melhem et al. (1976) al analizar el polen de 5 especies: $H$. brevispira, H. macropetala [H. brevispira], $H$. mollis, $H$. ovata y $H$. sacarolha y describen los granos como pilados, 3-porados, poros circulares, oblatos y de ámbito triangular; exina más gruesa alrededor de los poros, siendo la nexina más delgada que la sexina. Huang $(1967,1972)$ describe los granos de H. angustifolia; sus descripciones coinciden básicamente con las ante- riores, pero interpreta que la sexina es granulada. Chaudhuri (1969) en su contribución a la morfología polínica de las Sterculiaceae, incluye las especies $H$. hirsuta y $H$. javensis y considera que los granos son 3-colporados, brevicolpados y tectado-gemados.

Respecto a los otros géneros tratados en la literatura clásica dentro de la tribu Helictereae: Kleinhovia L., Neoregnellia Urb., Pterospermum Schreb., Reevesia Lindl., Veeresia Monach. et Moldenke y Ungeria Schott et Endl., la bibliografía sobre la morfología polínica es igualmente escasa y las descripciones están basadas en observaciones con microscopio óptico.

Zebe (1915) describe los granos de Pterospermum como esféricos, de tamaño grande, 50 $80 \mu \mathrm{m}, \mathrm{y}$ provistos de grandes aguijones obtusos y los de Kleinhovia, Helicteres, Reevesia y Ungeria, como granos pequeños, de 20 a 29 um; agrega además que los de Reevesia son tetraédricos, con los ángulos brevemente alargados en forma de cilindro hueco y con escultura finamente reticulada.

Rao (1950) menciona que Pterospermum, con granos esféricos y espinescentes, sería más afín a las Dombeyeae y que Kleinhovia, al igual que Helicteres, presenta granos triangulares y oblatos, pero se diferencia en que la exina es marcadamente granulada y la intina uniformemente delgada.

Erdtman (1966) describe los granos de Kleinhovia hospita y Neoregnellia cubensis como 3brevicolpor(oid)ados, angulaperturados, oblatos a peroblatos, con elementos negativos (OL- 
pattern) en el primero y elementos positivos (LO-pattern) en el segundo, y los de Reevesia pubescens como 4-5-brevicolporados, no angulaperturados, suboblatos y reticulados. Huang $(1967,1972)$ junto con $H$. angustifolia, describe los granos de Kleinhovia hospita, Reevesia formosana y Pterospermum acerifolium; señala que en las dos primeras especies la sexina es reticulada mientras que en Pterospermum es equinada-granulada y además los granos son de tamaño grande $(55-77 \mu \mathrm{m})$; al referirse a las aperturas el autor considera los granos como 3 -porados en las tres especies. Chaudhuri (1969) analiza el polen de Kleinhovia hospita y Reevesia pubescens, cuyos granos difieren entre sí principalmente en las aperturas y en la ornamentación de la exina: 3-zonopororados y con superficie granular en la primera y 4-5colporados y reticulados en la segunda.

Petrov \& Drazheva-Stamatova (1972) analizaron la morfología del polen de tres especies de Reevesia (R. pubescens Mart., R. thyrsoidea Lindl. y R. longipetiolata Merr. et Chun) con el fin de aportar el mayor número de caracteres cualitativos y cuantitativos que permitan la identificación de los granos de este género en sedimentos del Terciario; realizan una descripción detallada del polen de cada especie que básicamente coincide con la de Erdtman (op. cit.) y agregan que los granos pueden ser angulaperturados y que el retículo es heterobrocado y curvimurado.

En el presente estudio, al analizar la pared de los granos con microscopio electrónico de barrido, se aportan detalles de la escultura que amplían y, en el caso de Helicteres, modifican las descripciones anteriores.

\section{Maiteriales y métodos}

Se analiza el polen de 41 especies de Helicteres y con fines comparativos, el de los géneros monoespecíficos afines Neoregnellia, Kleinhovia y Ungeria y una especie de Reevesia, de Veeresia y de Pterospermun. De la mayoría de las especies de Helicteres, se analizaron dos ejemplares de distintas localidades.

El material polínico fue acetolizado de acuerdo a Erdtman (1966) y montado en gelatina glicerinada. Los preparados palinológicos se encuentran incluidos en la Palinoteca de la
Universidad Nacional del Nordeste (PALCTES). Para la observación con microscopio electrónico de barrido (MEB), se utilizaron granos acetolizados, que fueron deshidratados a través de una serie de alcoholes $\left(60^{\circ}-70^{\circ}-100^{\circ}\right)$, montados en una platina metálica y bañados con oro.

Las observaciones y descripciones se realizaron utilizando microscopio óptico (MO) Leitz Diaplan. De cada ejemplar se midieron, en un mínimo de 25 granos, la longitud del eje polar (P) y el diámetro ecuatorial (E) y, en un menor número de granos (10), diámetro del ectoporo y endoporo y espesor de la exina en apoporios, mesoporios y zona apertural. El análisis de la escultura de la exina se realizó sobre la base de fotomicrografías tomadas con el microscopio electrónico de barrido (MEB) Jeol JSM-580 OLV.

La terminología utilizada es básicamente la propuesta por Erdtman (1966) y Punt et al. (1994).

\section{Material estudiado de Helicteres:}

H. Andersonii Cristóbal: BRASIL. Goiás: Agua Fria, Hatschbach et al. 60225 (CTES), PAL-CTES 5531; ídem, Hatschbach et al. 60122 (CTES) PAL-CTES 5664.

$H$. angustifolia L.: MICRONESIA. Yap, Caroline Islands, Wong 537 (A), PAL-CTES 5564. VIET NAM. Hue and vicinity, Squires 147 (A), PAL-CTES 5577.

H. aspera A.St.-Hil. \& Naudin: BRASIL. Tocantins: $8 \mathrm{~km} \mathrm{~N}$ de Campos Belos, Cristóbal et al. 690 (CTES), PAL-CTES 5534.

H. baruensis Jacq.: BRASIL. Bahia: $44 \mathrm{~km}$ E de Ibotirama, Krapovickas et al. 38827 (CTES), PALCTES 5518; Pernambuco: Floresta, Andrade Lima s/n (CTES), PAL-CTES 5839. VENEZUELA. Zulia: entre Maracaibo y Urdaneta, Bunting et al. 11742 (CTES), PAL-CTES 5705.

H. biflexa Cristóbal: BRASIL. Bahia: Caetité, Arbo et al. 5648 (CTES), PAL-CTES 5667.

H. brevispira A.St.-Hil.: BOLIVIA. Beni, Vaca Diez, de Michel et al. 1272 (CTES), PAL-CTES 5699. BRASIL. Minas Gerais: Joaquim Felicio, Rossi SPF 23075 (CTES), PAL-CTES 5524; Tocantins: 7 km S de Dianópolis, Kraporickas et al. 37864 (CTES), PALCTES 5840. COLOMBIA. Cundinamarca: entre Nilo y la Porquera, Murillo et al. 377 (COL), PAL-CTES 5678. PARAGUAY. Amambay: $50 \mathrm{~km}$ SE de Bella Vista, Schinini et al. 20594 (CTES), PAL-CTES 5841.

H. carthagenensts Jacq.: COLOMBIA. Bolivar: W de Cartagena, Gentry et al. 47640 (CTES), PAL-CTES 5538. 
H. Cidin Cristóbal: BRASIL. Mato Grosso: São Féliz do Araguaia, Cld-Ferreira et al. 6484 (CTES), PAL-CTES 5666; Santa Terezinha, Cid-Ferreirn et al. 6383 (CTES), PAL-CTES 5697.

H. corylifolıa Nees \& Mart.: BRASIL. Goiás: 1 km E de Nazario, Krapovickas et al. 42916 (CTES), PALCTES 5529.

H. denticulenta Cristóbal: BRASIL. Goiás: 84 km NE de Formosa, Arbo et al. 3498 (CTES), PAL-CTES 5704; Mato Grosso: $40 \mathrm{~km} \mathrm{~S}$ de BR-070, camino a Poconé, Krapourckas et al. 43063 (CTES), PAL-CTES 5526.

H. Eichlern K. Schum.: BRASIL. Bahia: Itaberaba, Krapovickas et al. 38814 (CTES), PAL-CTES 5521; Pernambuco: Pombos, Krapovickas et al. 38015 (CTES), PAL-CTES 5694 - 5722; 6 km S de Arco Verde, Krapoutckas et al. 38076 (CTES), PAL-CTES 5721.

H. Eitenii Leane: BRASIL. Maranhão: Carolina, Krapovickas et al. 37849 (CTES), PAL-CTES 56965726; Tocantins: Mun. Tocantinópolis, Kraporickas et al. 37840 (CTES), PAL-CTES 5590; idem, Krapovickas et al. 37847 (CTES), PAL-CTES 5725.

H. elongata Wall. ex Mast.: BURMA. Maymyo Plateau, 28.VI.1913, Lace 4116 (A), PAL-CTES 5684. CHINA. Szemas, Henry 12218 (NY), PAL-CTES 5679.

H. Gardneriana A.St.-Hil. \& Naudin: BRASIL. Mato Grosso: $15 \mathrm{~km}$ de Poconé, Krapovickas et al. 43088 (CTES), PAL-CTES 5527. PARAGUAY. San Pedro: Jejui-Guazú, Krapovickas et al. 34270 (CTES), PAL- CTES 5700.

H. guazumifolia H.B.K.: BOLIVIA. Santa Cruz: Parque Nac. Kempff Mercado, Rodriguez et al. 691 (CTES), PAL-CTES 5680. CUBA. Pinar del Río: Loma Cayo Malo, Bisse et al. s/n (HAIB 38578), PALCTES 5688. MÉXICO. Tabasco: $18 \mathrm{mi}$ E de Villa Hermosa, Stervart et al. 84-8 (CTES), PAL-CTES 5585. VENEZUELA. Bolívar: Cedeño, Rutkıs 349 (CTES), PAL-CTES 5586; Cojedes: $12 \mathrm{~km} \mathrm{~S}$ de Santa Lucía, Ramua et al. 3518 (CTES), PAL-CTES 5787.

H. heptandra L.B.Sm.: BRASIL. Bahia: Mun. Rch. das Neves, Krapovickas et al. 38714 (CTES), PALCTES 5696; Ceará: Mun. Umirim, Valls et al. 11005 (CTES), PAL-CTES 5519.

H. hirsuta Lour.: CHINA. Hainan, 12.III.1934, Liang 65444 (NY), PAL-CTES 5562; idem, 15.X.1934, Liang 66284 (NY), PAL-CTES 5561. FILIPINAS. Culion Is., XII.1927, Ramos et al. 49931 (NY), PALCTES 5598.

H. isora L.: CHINA. Hainan, McClure 20137 (NY), PAL-CTES 5535. INDONESIA. Java, Buwwanda 7430 (A), PAL-CTES 5578.

H. jamaicensis Jacq.: ANTILLAS NEERLANDESAS. Inagua: Great Inagua, Dunbar 259 (GH), PAL-CTES 5686. REPÚBLICA DOMINICANA. La Vega, Jiménez 6199 (CTES), PAL-CTES 5594.
H. javensis Blume: INDONESIA. Java, Blume s/n (IAN), PAL-CTES 5683; ídem, Cult. Hort. Bogor., 1903 (GH), PAL-CTES 5565.

H. Krapoutckasii Cristóbal: BRASIL. Bahia: Formosa, Mendonça et al. 1360 (CTES), PAL-CTES 5701; Tocantins: 20 km E de Dianópolis, Krapovickas et al. 37866 (CTES), PAL-CTES 5540.

H. laciniosa Cristóbal: BRASIL. Bahia: Mun. Jandaíra, Queiroz 4672 (CTES), PAL-CTES 5541; ídem, Hatschbach et al. 63152 (CTES), PAL-CTES 5703.

H. Lanata (Teyjsm. \& Binn.) Kurz: TAILANDIA. Petchaboury, VI.1868, Pierre 3747 (A), PAL-CTES 5677.

H. lenta Mart.: BRASIL. Pará: Parque Indigena do Tumucumaque, Cavalcante 2589 (CTES), PALCTES 5589.

H. Lhotzkyana (Schott \& Endl.) K. Schum. var. Lhotzkyana: BRASIL. Mato Grosso: Mun. Cáceres, Krapovickas et al. 40201 (CTES), PAL-CTES 5723. PARAGUAY. Amambay: Cerro Corá, Schinin et al. 20321 (CTES), PAL-CTES 5532.

H. Lhotzkyana (Schott \& Endl.) K. Schum. var. pubinervis Kuntze: ARGENTINA. Salta: Depto. San Martín, Charpin et al. 22954 (CTES), PAL-CTES 5682; idem, Krapoutckas et al. 46332 (CTES), PAL-CTES 5724.

H. longepedunculata K. Schum.: BRASIL. Minas Gerais: Mun. Porteirinha, Taylor et al. 1498 (CTES), PAL-CTES 5592.

H. macropetala A. St.-Hil.: BRASIL. Bahia: $8 \mathrm{~km}$ de Mirangaba, Arouck Ferretra 65 (CTES), PAL-CTES 5698; Pernambuco: Mun. Arco Verde, Krapovickas et al. 38056 (CTES), PAL-CTES 5530.

H. muscosa Mart.: BRASIL. Ceará: Missão Velha, Valls et al. 10925 (CTES), PAL-CTES 5692; Pará: Serra do Cachimbo, Kırkbride 2789 (MO), PAL-CTES 5665; Piauí: $15 \mathrm{~km} \mathrm{~S}$ de Jaicos, Krapovnckas et al. 38784 (CTES), PAL-CTES 5520.

H. ovata Lam.: BRASIL. Minas Gerais: $18 \mathrm{~km} \mathrm{~N}$ de Ouro Preto, Krapovickas et al. 33470 (CTES), PALCTES 5533 - 5838; Rio de Janeiro: Rio de Janeiro, Restinga do Grumari, Sucre 3361 (RB), PAL-CTES 5691.

H. pentandra L.: BRASIL. Rondônia: Mun. Ariquemes, Frame et al. 116 (CTES), PAL-CTES 5539. GUAYANA FRANCESA. Cachoeira Camarauá, Irvin et al. 48543 (CTES), PAL-CTES 5695.

H. Pilgeri R.E.Fr.: BRASIL. Mato Grosso: $130 \mathrm{~km}$ $\mathrm{N}$ de Barra do Garças, Krapouıckas et al. 42962 (CTES), PAL-CTES 5528.

H. Pentonis Cristóbal: BRASIL. Bahia: $55 \mathrm{~km}$ de Vitória da Conquista, Brazão 234 (HRB), PAL-CTES 5681.

H. Rekoi Standl.: MÉXICO. Guerrero: Mun. La Unión, Koch et al. 83121 (CTES), PAL-CTES 5537. 
H rufipila Cristóbal. BRASIL. Bahia' Mun. Abaíra, Ganev 1317 (CTES), PAL-CTES 5593.

H. sacarolha A.St.-Hil., A. Juss. \& Cambess.: BRASIL. Goiás: Caldas Novas, Menezes, SPF 22582 (CTES), PAL-CTES 5523.

H. semitriloba Bertero ex DC.: CUBA. Honguín: Sagua de Tánamo, Bisse et al. s/n (HAJB 43004), PALCTES 5689. REPÚBLICA DOMINICANA. Valverde: $\mathrm{N}$ de Guayacanes, Clcero et al. 6378-B (CTES), PALCTES 5584.

H. trapezifolia A. Rich.: CUBA. Honguin: North slope of Sierra Nipe, Morton et al. 2985 (US), PALCTES 5595; Pinar del Río: Cajálbana, Alain et al. 1382 (NY), PAL-CTES 5690.

H. Vegae Cristóbal: MÉXICO. Sinaloa: Mun. Mocorito, Vega et al. 2074 (CTES), PAL-CTES 5536.

H. velutma K. Schum.: BRASIL. Bahia: Mun. Caetité, Carvalho et al. 1768 (CTES), PAL-CTES 5702; Minas Gerais: Vale do rio Itacambiruçu, Semur et al., SPF 42924 (CTES), PAL-CTES 5525.

H. viscida Blume: CHINA. Hainan, Dung Ka to Wen Fa Shi, 1932-33, Chun et al. 44283 (NY), PALCTES 5563. VIET NAM. Annam, Vinh. XI.1920, Poilane 2430 (A), PAL-CTES 5685.

H. vuarame Mart. emend. Cristóbal: BRASIL. Bahia: Monte Santo, Quetroz 4587 (CTES), PAL-CTES 5566; Pernambuco: Rusinha, Krapoutckas et al. 38006 (CTES), PAL-CTES 5522.

\section{Descripción de los granos de polen de Helicteres}

\section{Caracteres generales:}

Granos 3-zonoporados, con menor frecuencia 4-zonoporados. Estos últimos generalmente se presentan en porcentajes bajos (2-5\%), excepto en $H$. baruensis (Bunting 11742) y $H$. hirsuta (Liang 65444) donde alcanzan un 17 y $30 \%$, respectivamente. Isopolares y radiosimétricos, a veces algo asimétricos, por la disposición no equidistante de los poros. En $H$. Lhotzkyana var. Lhotzkyana (Schinini 20321), además de los granos normales (3-4 porados) se observaron granos anormales, asimétricos y con número reducido de aperturas (2 porados, con ámbito elíptico, $6 \%$, o con ámbito triangular, $9 \%$, y 3 porados con ámbito cuadrangular, 23\%).

En general, son oblatos o suboblatos, rara vez oblato-esferoidales o peroblatos, con contorno elíptico. Ámbito triangular o cuadran- gular, angulaperturado; mesoporios rectos, levemente convexos o levemente cóncavos.

En cuanto al tamaño son de pequeños a medianos, los valores medios del eje polar (P) y diámetro ecuatorial (E) oscilan entre 14,2-27,5 $\mu \mathrm{m}$ y $22,7-37,5 \mu \mathrm{m}$, respectivamente. Los granos de menor tamaño se encuentran en $H$. Gardneriana y $H$. rufipila y los más grandes en H. carthagenensis (Tabla 1).

Poros circulares o levemente alargados en sentido meridional, endoporo de mayor diámetro que el ectoporo; rodeando al poro puede diferenciarse una zona anular (anillo) generalmente psilada.

Exina de 0,7-1,7 $\mu \mathrm{m}$ de espesor en apoporios y mesoporios, engrosada alrededor de las aperturas $(1,4-5 \mu \mathrm{m})$. Sexina igual o más gruesa que la nexina, tectada-columelada; las columelas son especialmente visibles en las cercanías de los poros, la altura de las mismas disminuye notablemente en los mesoporios; con elementos supratectales de tamaño y distribución variables. Nexina igual o más delgada que la sexina, con frecuencia poco diferenciada y generalmente muy irregular.

El microscopio electrónico de barrido reveló que la sexina es, en la mayoría de las especies, tectado-perforada, con perforaciones diminutas, del orden de 0,10 um de diámetro o con fósulas, que pueden llegar a alcanzar más de $1 \mu \mathrm{m}$ de largo; con frecuencia se anastomosan entre sí, a tal grado que dejan fragmentos aislados de exina (micro-rúgulas). En $H$. Rekoi, es microrreticulada y en $H$. carthagenensis, tectada con escasas perforaciones. En la zona que rodea a los poros es pertectada (psilada o microfoveolada).

Se pueden reconocer 3 tipos de elementos esculturales supratectales: báculas, microespinas y verrugas. Estos últimos pueden ser desde bien definidos hasta muy atenuados, gradación que culmina en una superficie totalmente lisa.

La ornamentación puede ser más o menos uniforme en toda la superficie del grano o bien presentar una clara diferenciación entre las áreas polares y la zona ecuatorial. En el primer caso, el anillo o zona anular que rodea al poro es bien definido y carece de ornamentación, mientras que, en el segundo caso no está diferenciado, 
TABLA 1: Tipos polínicos y dimensiones, en micrómetros, del eje polar (P), diámetro ecuatorial (E); espesor de la exina en apoporios (Ap) y mesoporios (Msp) y alrededor de las aperturas (Apt) y diámetro de endoporos y exoporos, de las especies estudiadas de Helicteres.

\begin{tabular}{|c|c|c|c|c|c|c|c|c|}
\hline $\begin{array}{c}\text { Secciones } \\
y \\
\text { Especies }\end{array}$ & $\begin{array}{l}\text { Tipos } \\
\text { polínicos }\end{array}$ & $\begin{array}{c}P \\
\text { media (rango) }\end{array}$ & $\begin{array}{c}E \\
\text { media (rango) }\end{array}$ & $\begin{array}{c}\mathrm{P} / \mathrm{E} \\
\text { media (rango) }\end{array}$ & \multicolumn{2}{|c|}{ Espesor de la exina } & $\begin{array}{l}\text { Diámetr } \\
\text { Exoporo }\end{array}$ & Endoporo. \\
\hline \multicolumn{9}{|l|}{ Secc. HELICTERES } \\
\hline H. isora & TIPO I & $18,2(15,0-21,0)$ & $23,2(18,5-28,7)$ & $0,77(0,70-0,85)$ & $1,0-1,4$ & $2,0-3,5$ & $1,4-2,0$ & $2,8-4,0$ \\
\hline \multicolumn{9}{|c|}{ Secc. ORTHOCARPAEA DC. } \\
\hline H. angustifolia & TIPO II & $21,0(16,8-22,8)$ & $26,0(22.4-28,7)$ & $0.78(0.72-0.85)$ & 1,0 & 2,8 & $3,5-4,0$ & $4,5-6,0$ \\
\hline H. elongata & TIPO II & $23,0(20,3-25,5)$ & $30,3(24,5-33,0)$ & $0,76(0,63-0,85)$ & $1.0-1.2$ & $2,0-2,5$ & $5,0-7,0$ & $6,0-7,8$ \\
\hline H. lanata & TIPO II & $20,0(19,0-21,0)$ & $25,8(24,5-27,5)$ & $0,77(0.71-0,83)$ & 1.0 & $2,3-2,8$ & 2,0 & 3,5 \\
\hline H. javensis & TIPO II & $19,4(18,2-21,7)$ & $23,4(21,2-25,2)$ & $0,82(0,73-0,86)$ & 1,0 & $2,0-3,0$ & $5,0-6,0$ & $7,0-8,0$ \\
\hline H. hirsuta & TIPO VI & $20,2(16,8-26,6)$ & $27,7(24,5-35,0)$ & $0,72(0,65-0,81)$ & $1,0-1,3$ & $2,4-3,5$ & $2,0-2,5$ & $3,5-5,0$ \\
\hline H. viscida & TIPO VI & $18,4(17,5-19,6)$ & $25,6(22,4-28,0)$ & $0.71(0.67-0.78)$ & $1,0-1.4$ & $2.8-4,2$ & $2,0-3,3$ & $3.0-4,5$ \\
\hline \multicolumn{9}{|c|}{ Secc. STEGOGAMOS Cristóbal } \\
\hline H. Vegae & TIPO III & $21,4(19,6-22,4)$ & $29,0(26,0-33,0)$ & $0,74(0,65-0,82)$ & $1,0-1,5$ & $1,4-2,9$ & $3,0-5,0$ & $5,0-7,0$ \\
\hline \multicolumn{9}{|c|}{ Secc. POLYANDRIA Cristóbal } \\
\hline H. Rekoi & TIPO IV & $2 \mathrm{I}, 5(19,0-24,5)$ & $30,6(26,0-33,0)$ & $0,70(0,65-0,74)$ & 1,0 & $2,0-2,4$ & $3,0-3,5$ & 4,0 \\
\hline \multicolumn{9}{|c|}{ Secc. ALICTERES (Schott et Endl.) Cristóbal } \\
\hline $\mathrm{H}$ carthagenensis & TIPO V & $27,5(\overline{21,7}-34,3)$ & $37,5(30, \overline{0}-44,5)$ & $0,73(0,64-0,80)$ & $1,4-1,7$ & $3,0-4,9$ & 3.5 & 5,0 \\
\hline \multicolumn{9}{|c|}{ Secc. ORTHOTHECIUM (C Presl) Cristóbal } \\
\hline H. jamaicensis & TIPO VI & $28,3(26,0-31,5)$ & $35,6(34,0-38,5)$ & $0,79(0,76-\overline{0,81})$ & $1,0-2,0$ & $2,5-3,0$ & 3,5 & 7,0 \\
\hline H. laciniosa & TIPO VI & $24,2(21,0-27,3)$ & $31,0(28.0-33,6)$ & $0,77(0,68-0,85)$ & $1,0-1,4$ & $2,8-3,5$ & $2,8-5,0$ & $5,6-8,0$ \\
\hline H. semitriloba & TIPO VI & $19,3(16,0-21,0)$ & $27,0(25,2-29,4)$ & $0,71(0,60-0,77)$ & $0,7-1,4$ & $2,0-2.8$ & $2.8-3.5$ & $4,0-5,0$ \\
\hline H. trapezifolia & TIPO VI & $19,9(18,0-21,0)$ & $27,2(24,5-30,0)$ & $0,73(0,64-0,85)$ & 1,0 & 2,5 & $1,5-2,5$ & $2,0-3,5$ \\
\hline H. baruensis & TIPO VII & $20,6(16,0-25,2)$ & $28,6(24,5-35,0)$ & $0.70(0.62-0.80)$ & $1,0-1,5$ & $2,0-3,0$ & $2,8-3,5$ & $4,2-5,0$ \\
\hline $\begin{array}{l}\text { H. Lhotzkyana } \\
\text { var. Lhotzkyana }\end{array}$ & TIPO VII & $18,8(17,5-22,0)$ & $26,0(22,5-29,4)$ & $0,72(0,65-0,83)$ & $1,0-1,2$ & $2,0-2,4$ & $1,6-2,0$ & 5,0 \\
\hline $\begin{array}{l}\text { H. Lhotzkyana } \\
\text { var pubinervis }\end{array}$ & TIPO VII & $18,0 \overline{(16,5-20,0)}$ & $25,0(20,0-28,5)$ & $0,73(0,70-0,77)$ & $1,0-\overline{1,2}$ & $2,0-2,5$ & 3,0 & 5,0 \\
\hline H. vuarame & TIPO VII & $24,7(21,0-26,6)$ & $32,5(26,6-37,8)$ & $0,76(0,71-0,90)$ & $1,0-1,7$ & $1,6-3,0$ & $2,5-2,8$ & 3,5 \\
\hline H. brevispira & TIPO VIII & $16,2(12.6-18,6)$ & $24,0(21,0-27,3)$ & $0,65(0,58-0,80)$ & $1,0-2,0$ & $1,2-2,6$ & $1,5-2,0$ & $3,5-4,0$ \\
\hline
\end{tabular}


TABLA 1(cont.): Tipos polínicos y dimensiones, en micrómetros, del eje polar (P), diámetro ecuatorial (E); espesor de la exina en apoporios (Ap) y mesoporios (Msp) y alrededor de las aperturas (Apt) y diámetro de endoporos y exoporos, de las especies estudiadas de Helicteres.

\begin{tabular}{|c|c|c|c|c|c|c|c|c|}
\hline H. macropetala & TIPO VIII & $16,2(14,7-18,0)$ & $26,6(23,8-30,0)$ & $0,61(0,53-0,71)$ & $1,0-1,4$ & $1,5-2,1$ & $1,5-2,0$ & $2,5-4,2$ \\
\hline H. nufipila & TIPO VIII & $14,2(13,0-17,0)$ & $27,5(24,5-30,0)$ & $0.51(0,48-0,64)$ & 1,0 & 2,2 & 1,5 & 3,0 \\
\hline H. Andersonii & TIPO IX & $19,5(17,5-24,5)$ & $24,2(21,0-28,0)$ & $0,80(0,71-0,91)$ & $1,0-1,4$ & $2,8-3,5$ & $1,5-2,0$ & $2,0-4,0$ \\
\hline H. biflexa & TIPO IX & $20,8(19,0-22,4)$ & $26,6(24,5-28,0)$ & $0.78(0,71-0,83)$ & $1,0-1,2$ & $2,0-2,4$ & 2,8 & 3,5 \\
\hline H. longepedunculata & TIPO [X & $16,5(15,5-18,2)$ & $23,2(21,5-24,5)$ & $0,71(0,65-0,79)$ & $1,0-1,2$ & $2,0-2,4$ & $1,5-2,0$ & $2,0-4,0$ \\
\hline H. ovata & TIPO IX & $22,5(20,3-25,2)$ & $31,0(28,7-36,5)$ & $0,72(0,63-0,84)$ & $1,0-2,0$ & $2,2-4,4$ & $2,0 \cdot 2,8$ & $2,8-4,5$ \\
\hline H. velutina & TIPO IX & $16,7(14,5-25,2)$ & $24,0(21,0-25,2)$ & $0,69(0,61-0,82)$ & $1,0-1,4$ & $2,4-2,8$ & $2,0-2,8$ & $4,2-5,0$ \\
\hline \multicolumn{9}{|c|}{ Secc. SACAROLHA K Schum. } \\
\hline H. aspera & TIPOIX & $22,3(18,9-24,5)$ & $28,5(26,6-30,8)$ & $0,78(0,71-0,84)$ & 1,2 & $2,0-2,4$ & $2,8-3,5$ & $5,0-6,3$ \\
\hline H. Cidii & TIPO IX & $21,2(20,3-22,5)$ & $27,3(26,0-28,0)$ & $0,77(0,73-0,81)$ & $1,0-1,5$ & $2,0-2,5$ & 2,0 & 5,0 \\
\hline H corylifolia & TIPO IX & $18,6(16,8-22,4)$ & $24,3(22,0-25,9)$ & $0.77(0,68-0,86)$ & $1,0-1,5$ & 2,5 & $2,0-2,8$ & $2,8-4,0$ \\
\hline H. denticulenta & TIPO IX & $20,8(16,8-23,8)$ & $26,2(24,5-28,0)$ & $0,79(0.68-0.91)$ & $0,7-1,4$ & $1,5-3,5$ & $2,0-2,8$ & $3,5-5,0$ \\
\hline H. Eichleri & TIPO IX & $16,6(15,5-19,5)$ & $23,8(21,0-26,0)$ & $0,69(0,61-0,80)$ & $1,0-1,4$ & $2,0-3,5$ & $2,0-2,5$ & $3,0-3,5$ \\
\hline H. Eitenii & TIPO IX & $20,3(17,5-24,5)$ & $27,0(25,2-30,0)$ & $0,74(0,62-0,85)$ & $1,0-1,5$ & $2,5-3,5$ & $2,5-3,0$ & 5,0 \\
\hline H. Gardneriana & TIPO IX & $17,7(16,0-19,0)$ & $22,7(21,7-24,2)$ & $0,78(0,73-0,84)$ & $1,0-1,2$ & $2,0-2,6$ & $1,5-2,5$ & $2,5-3,5$ \\
\hline H. guazumifolia & TIPO IX & $20,2(16,0-23,8)$ & $26,0(23,8-29,4)$ & $0.77(0,65-0,91)$ & $1,0-1,4$ & $2,4-3,5$ & $2,8-3,5$ & $4,2-6,0$ \\
\hline H. heptandra & TIPO IX & $17,6(16,0-23,6)$ & $23,8(22,0-27,3)$ & $0,74(0,67-0,79)$ & $1,0-1,4$ & $1,4-2,4$ & $2,5-3,5$ & $4,2-5,0$ \\
\hline H. Krapovickasii & TIPO IX & $18,3(14,7-21,5)$ & $25,5(20,3-28,0)$ & $0,71(0,63-0.81)$ & $1,0-2,0$ & $2,0-3,0$ & $2,8-3,5$ & 5,0 \\
\hline H. lenta & TIPO IX & $21,3(21,0-22,4)$ & $28,2(26,0-29,4)$ & $0,75(0,71-0,82)$ & $1,0-1,4$ & 2,5 & $2,5-4,0$ & $3,5-6,3$ \\
\hline H. muscosa & TIPO IX & $18,9(16,5-21,7)$ & $25,8(23,0-28,0)$ & $0,73(0,65-0,81)$ & $1,0-2,1$ & $2,0-2,5$ & $2,0-3,0$ & $3,5-5,0$ \\
\hline H. pentandra & TIPO IX & $19,2(16,8-20,8)$ & $26,5(21,7-29,4)$ & $0,72(0,59-0,88)$ & $1,0-1,7$ & $2,0-3,7$ & $2,0-3,5$ & $4,0-5,5$ \\
\hline H. Pilgeri & TIPO IX & $18,7(17.5-20,3)$ & $26,0(23,8-28,0)$ & $0,72(0,64-0,83)$ & 1,2 & 2,4 & $2,0-3,5$ & $3,0-7,0$ \\
\hline H. Pintonis & TIPO IX & $20,4(19,5-21,7)$ & $28,3(27,0-31,5)$ & $0,72(0,69-0,77)$ & 1,2 & 2,5 & 2,8 & 3,5 \\
\hline H. sacarolha & TIPO IX & $20,2(17,5-23,0)$ & $26,9(24,5-29,4)$ & $0,75(0,67-0,84)$ & $1,0-1,2$ & $2,7-3,0$ & $2,0-3,0$ & $4,0-6,0$ \\
\hline
\end{tabular}


de manera que en granos ornamentados los elementos esculturales, aunque muy atenuados, llegan hasta el borde de las aperturas.

\section{Descripción de los tipos polínicos:}

Teniendo en cuenta los caracteres de la exina se pueden reconocer 9 tipos polínicos. El ordenamiento de los tipos polínicos sigue la secuencia que se presenta en la aparición de los distintos elementos esculturales supratectales y en una mayor complejidad de las perforaciones, la cual se puede interpretar como una especialización adaptativa para la función harmomégata. Se consideran como más simples los tipos polínicos que presentan una escultura uniforme en todo el grano y más complejos o "avanzados", los que presentan diferenciación entre las zonas polares y la ecuatorial.

Tipo I (Fig.1, A-B):

Se presenta únicamente en H. isora (secc. Helicteres).

Tectado-perforado, con báculas supratectales dispersas en toda la superficie. Perforaciones circulares distribuidas uniformemente en toda la supe:ficie, excepto alrededor de los poros. Báculas de hasta $1 \mu \mathrm{m}$ de altura, distanciadas 1-3 $\mu \mathrm{m}$ entre sí.

Ámbito triangular, con mesoporios levemente convexos. Poros circulares, de 2-3,5 $\mu \mathrm{m}$ de diámetro, con anillo psilado.

\section{Tipo II (Fig. 1, C-F):}

Especie característica $H$. angustifolia. Se presenta también en $H$. elongata, $H$. lanata y $H$. javensis (secc. Orthocarpaea).

Tectado-perforado, psilado o con verrugas supratectales muy atenuadas. Perforaciones usualmente circulares, uniformemente distribuidas. En $H$. lanata y $H$. javensis, la superficie es siempre psilada, en cambio en $H$. angustifolia y H. elongata, hay granos totalmente psilados y otros con verrugas débilmente marcadas, de distribución irregular.

Ámbito triangular con mesoporios convexos o levemente cóncavos o rectos en $H$. elongata.
Poros circulares de 2-7 $\mu \mathrm{m}$ de diámetro, anillo poco diferenciado.

\section{Tipo III (Fig. 2, A-B):}

Se presenta únicamente en H. Vegae (secc. Stegogamos).

Tectado-perforado, con verrugas supratectales localizadas principalmente en los mesoporios, mientras que las zonas polares son psiladas. La superficie del téctum, en mesocolpios, es densamente perforada y las verrugas miden, aproximadamente, $1 \mu \mathrm{m}$ de ancho $\times 0,5 \mu \mathrm{m}$ de alto. Hacia los polos, disminuye el número de perforaciones y las verrugas se atenúan hasta desaparecer.

Ámbito triangular, con mesoporios rectos. Poros alargados en sentido meridional, de 2,83,5 x 4-6 $\mu \mathrm{m}$, a veces circulares, con anillo psilado.

Tipo IV (Fig. 2, C-D):

Se presenta únicamente en $H$. Rekoi (secc. Polyandria).

Microrreticulado con verrugas supratectales en toda la superficie. Microrretículo formado por lúmenes circulares o alargados, de $(0,1) 0,5$ $(0,8) \mu \mathrm{m}$ de diámetro y muros de 0,15-0,3 $\mu \mathrm{m}$ de ancho; verrugas de aproximadamente 0,3$0,8 \mu \mathrm{m}$ de diámetro basal, distribuidas irregularmente.

Ámbito triangular con mesoporios rectos a levemente cóncavos. Poros circulares, de 3-3,5 $\mu \mathrm{m}$ de diámetro, con anillo psilado.

\section{Tipo V (Fig. 2, E-F):}

Se presenta únicamente en $H$. carthagenensis (secc. Alicteres).

Tectado, escábrido, con microverrugas supratectales tanto en apoporios como mesoporios. Verrugas de aproximadamente 0,5-1 $\mu \mathrm{m}$ de diámetro, densamente dispuestas. Puede presentar perforaciones aisladas.

Ámbito triangular, mesoporios levemente convexos. Poros alargados en sentido meridional, de 2-3,5 x 3-6 $\mathrm{m}$, con anillo psilado. 
Tipo VI (Fig. 3, A-D):

Especie característica H. semitriloba. Se presenta en $H$. hirsuta, $H$. viscida (secc. Orthocarpaea), $H$. jamaicensis, $H$. laciniosa, $H$. semitriloba y H. trapezifolia (secc. Orthothecium).

Tectado-perforado con verrugas supratectales en toda la superficie. Perforaciones generalmente circulares, densamente distribuidas en toda la superficie. Verrugas de $1 \mu \mathrm{m}$ o menos de diámetro basal y de contorno circular.

Ámbito triangular, con mesoporios rectos a levemente convexos. Poros circulares, de 2-3,5 um de diámetro, con anillo psilado.

\section{Tipo VII (Fig. 3, E-F; Fig. 4, A-B):}

Especie característica H. vuarame. Se presenta, además, en $H$. baruensis, H. Lhotzkyana var. Lhotzkyana y H. Lhotzkyana var. pubinervis (secc. Orthothecium).

Tectado-perforado, fosulado, con verrugas supratectales en toda la superficie. Perforaciones y fósulas (formadas por fusión de varias perforaciones dispuestas linearmente) distribuidas en toda la superficie. Verrugas de 1-1,5 $\mu \mathrm{m}$ de diámetro, a veces anastomosadas en grupos de 2-4. En H. Lhotzkyana var. Lhotzkyana las verrugas pueden encontrarse casi totalmente soldadas entre sí en los mesoporios (Fig. 4, B), de modo que la superficie es casi lisa en corte óptico ecuatorial.

Ámbito triangular, con mesoporios rectos a levemente convexos. Poros circulares, de 2-3,5 $\mu \mathrm{m}$ de diámetro, con anillo psilado.

\section{Tipo VIII (Fig. 4, C-F):}

Especie característica $H$. brevispira. Se presenta además en $H$. macropetala y $H$. rufipila (secc. Orthothecium).

Tectado a tectado-perforado, fosulado, parcialmente micro-equinado-verrugado. Las perforaciones y fósulas están concentradas en las áreas polares, de modo que los mesoporios son tectados, lisos o levemente escabrados. Las fósulas con frecuencia se fusionan entre sí (Fig. 4, D), hasta el extremo de dejar fragmentos ais- lados de exina muy irregulares (microrúgulas). En los polos se localizan, también, los procesos esculturales, del orden de $0,5 \mu \mathrm{m}$, que son una combinación de microverrugas y microespinas, densamente dispuestos.

Ámbito triangular, con mesoporios rectos a levemente cóncavos. Poros circulares, de 1,5-2 $\mu \mathrm{m}$ de diámetro o alargados en sentido meridional $(2 \times 3 \mu \mathrm{m})$ en $H$. macropetala; sin anillo diferenciado.

\section{Tipo IX (Fig. 5, A-F; Fig. 6, A-F):}

Especie característica H. sacarolha. Se presenta en 16 de las 18 especies de la secc. Sacarolha que han podido ser estudiadas y en algunas especies de la secc. Orthothecium (H. Andersonii, $H$. biflexa, $H$. longepedunculata, $H$. ovata y $H$. velutina).

Tectado-perforado, fosulado, microequinado en toda la superficie del grano. En la zona ecuatorial, el téctum es casi completo, foveolado y/o con perforaciones diminutas (punctas) y las microespinas son muy pequeñas o apenas insinuadas y llegan hasta el borde de los poros.

En las zonas polares las microespinas son más evidentes; igualmente, las perforaciones y fósulas. Estas últimas presentan una gran variación en cuanto a forma y tamaño: pueden ser lineares, sinuosas, ramificadas, cortas o largas, angostas o muy abiertas, oscilan entre 0,1 a casi $1 \mu \mathrm{m}$ de ancho $\times 0,2$ a más de $2 \mu \mathrm{m}$ de largo. En H. Andersonii, H. Cidii y $H$. Eitenii, hay predominio de perforaciones circulares, mientras que, en $H$. aspera, $H$. Eichleri, $H$. heptandra, H. Pintonis y H. velutina, predominan las fósulas que, además, pueden llegar a estar casi totalmente fusionadas entre sí. El resto de las especies presenta una variabilidad intermedia entre estos dos extremos. Estos caracteres no tienen valor diagnóstico para diferenciar las especies, debido a que la variabilidad puede presentarse aún dentro de un mismo especimen.

Ámbito triangular con mesoporios levemente convexos. Poros circulares, de 1,5-3,5 $\mu \mathrm{m}$ de diámetro, sin anillo. 

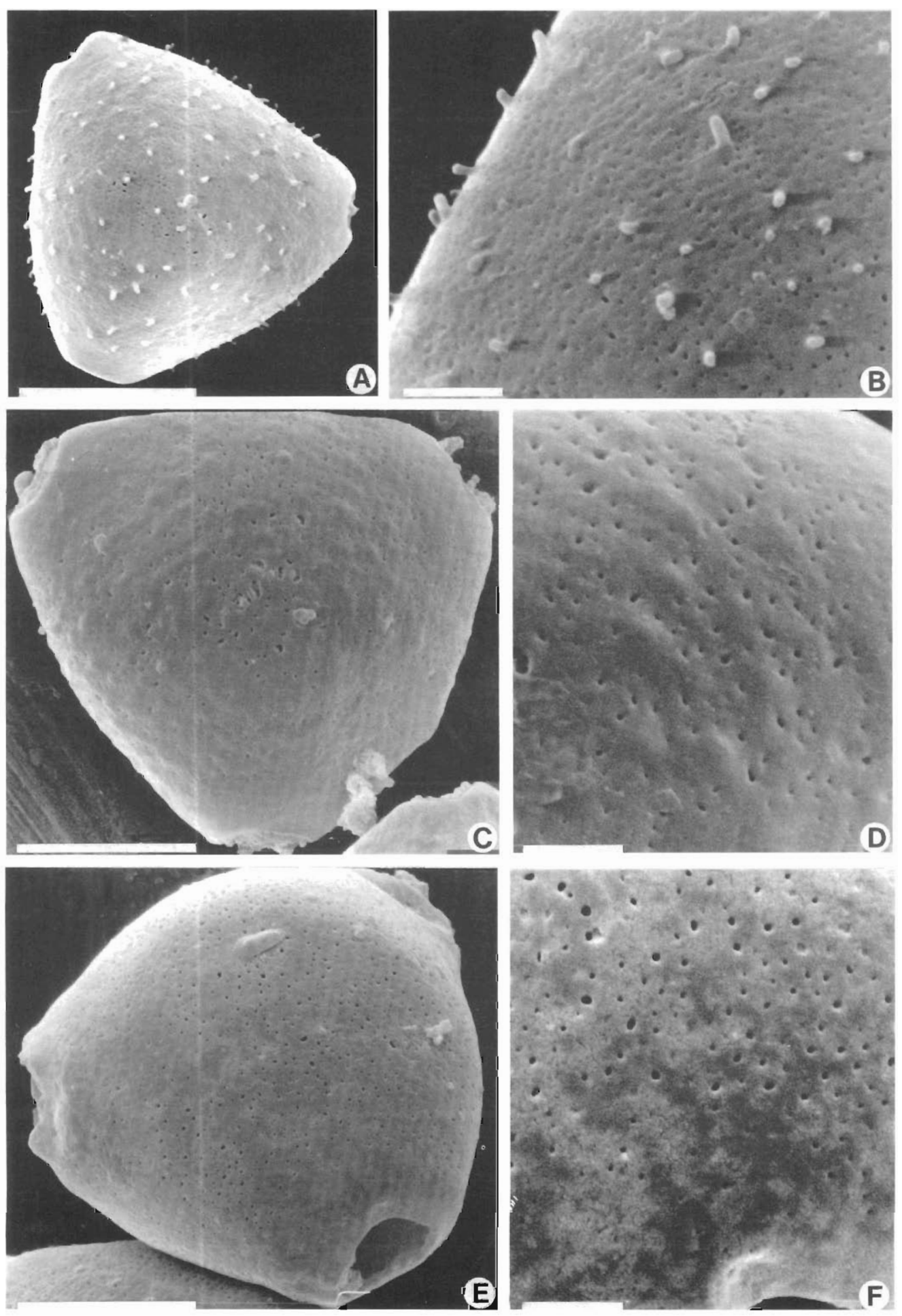

Fig. 1. Tipo I: A-B,Helicteres isora (McClure 20137). Tipo II: C-D,H. angustıfolia (Wong 537); E-F,H.javensis (Cult.Hort.Bogor. 1903). Escalas $=10 \mu \mathrm{m}(\mathrm{A}, \mathrm{C}, \mathrm{E}) ; 2 \mu \mathrm{m}(\mathrm{B}, \mathrm{D}, \mathrm{F})$. 

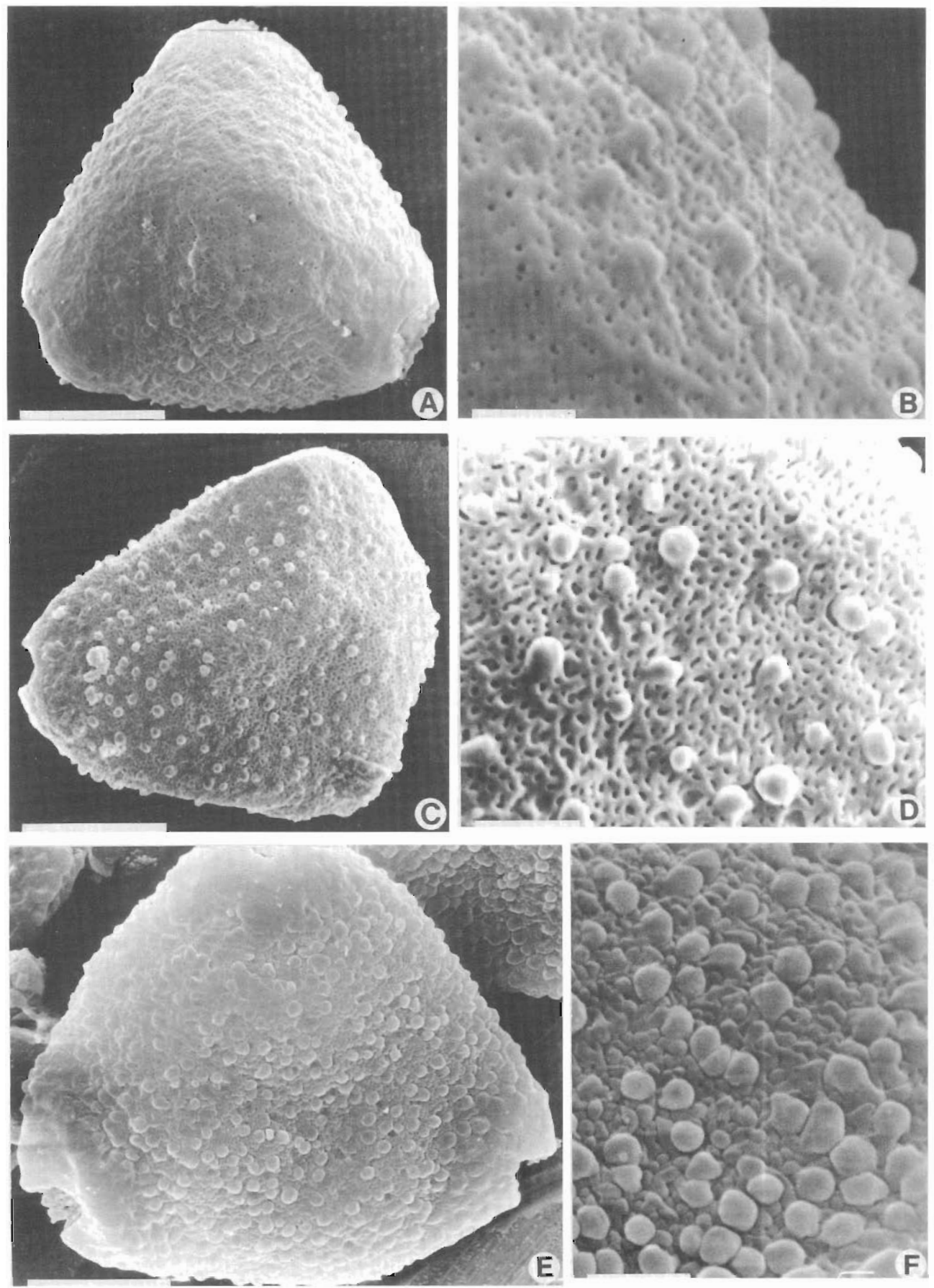

Fig. 2. Tipo III: A-B,Helicteres Vegae(Vegaetal. 2074). Tipo IV: C-D,H.Rekoi (Kochetal. 83121). Tipo V: E-F, H. carthagenensis (Gentry et al. 47640). Escalas $=10 \mu \mathrm{m}(\mathrm{A}, \mathrm{C}, \mathrm{E}) ; 2 \mu \mathrm{m}(\mathrm{B}, \mathrm{D}, \mathrm{F})$. 

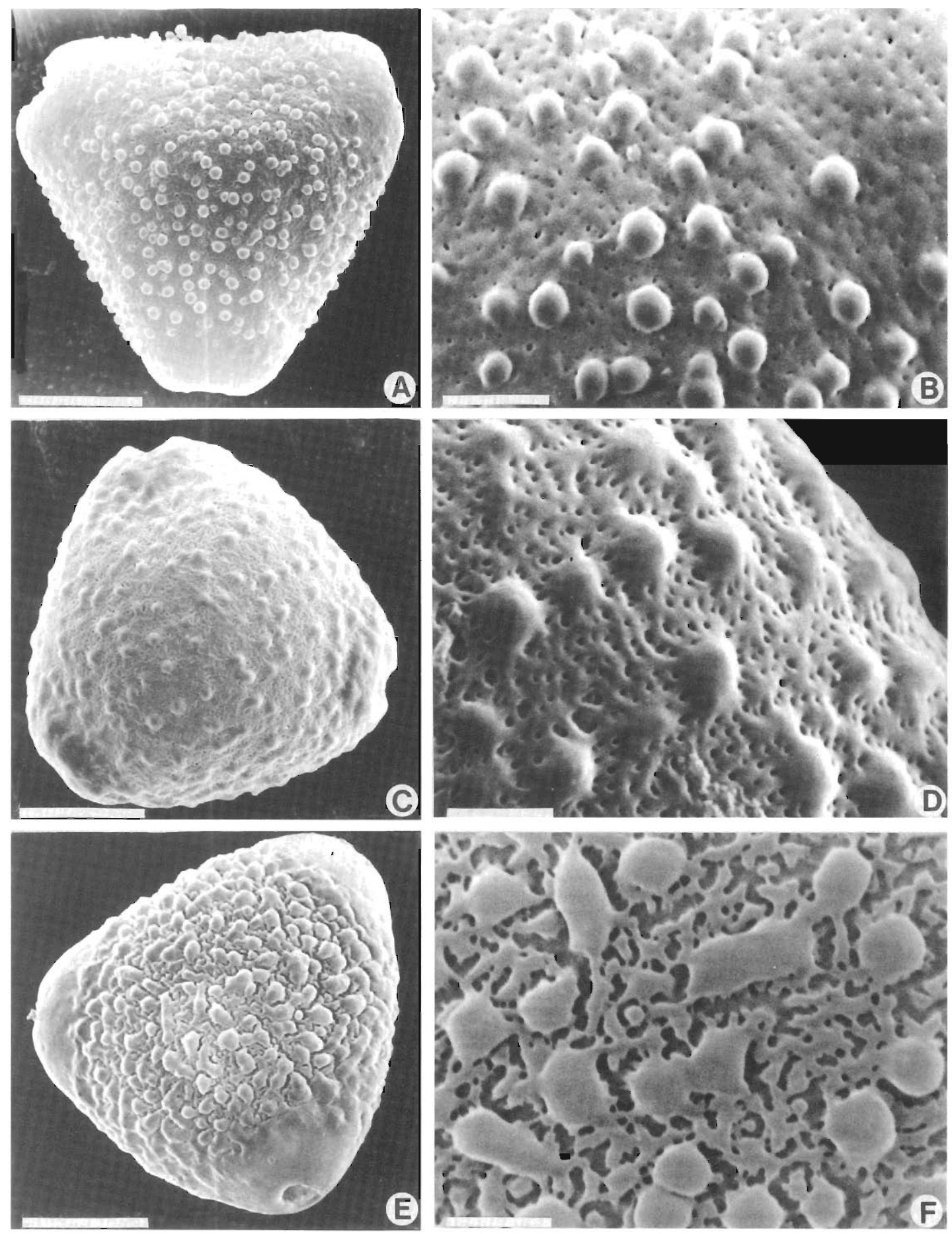

Fig. 3. Tipo VI: A-B, Helcteres semitriloba (Cicero et al. 6378-B); C-D, H. hirsuta (Liang 65444). Tipo VII: E-F, H. vuarame (Krapovickas et al. 38006). Escalas=10 $\mu \mathrm{m}(\mathrm{A}, \mathrm{C}, \mathrm{E}) ; 2 \mu \mathrm{m}(\mathrm{B}, \mathrm{D}, \mathrm{F})$. 

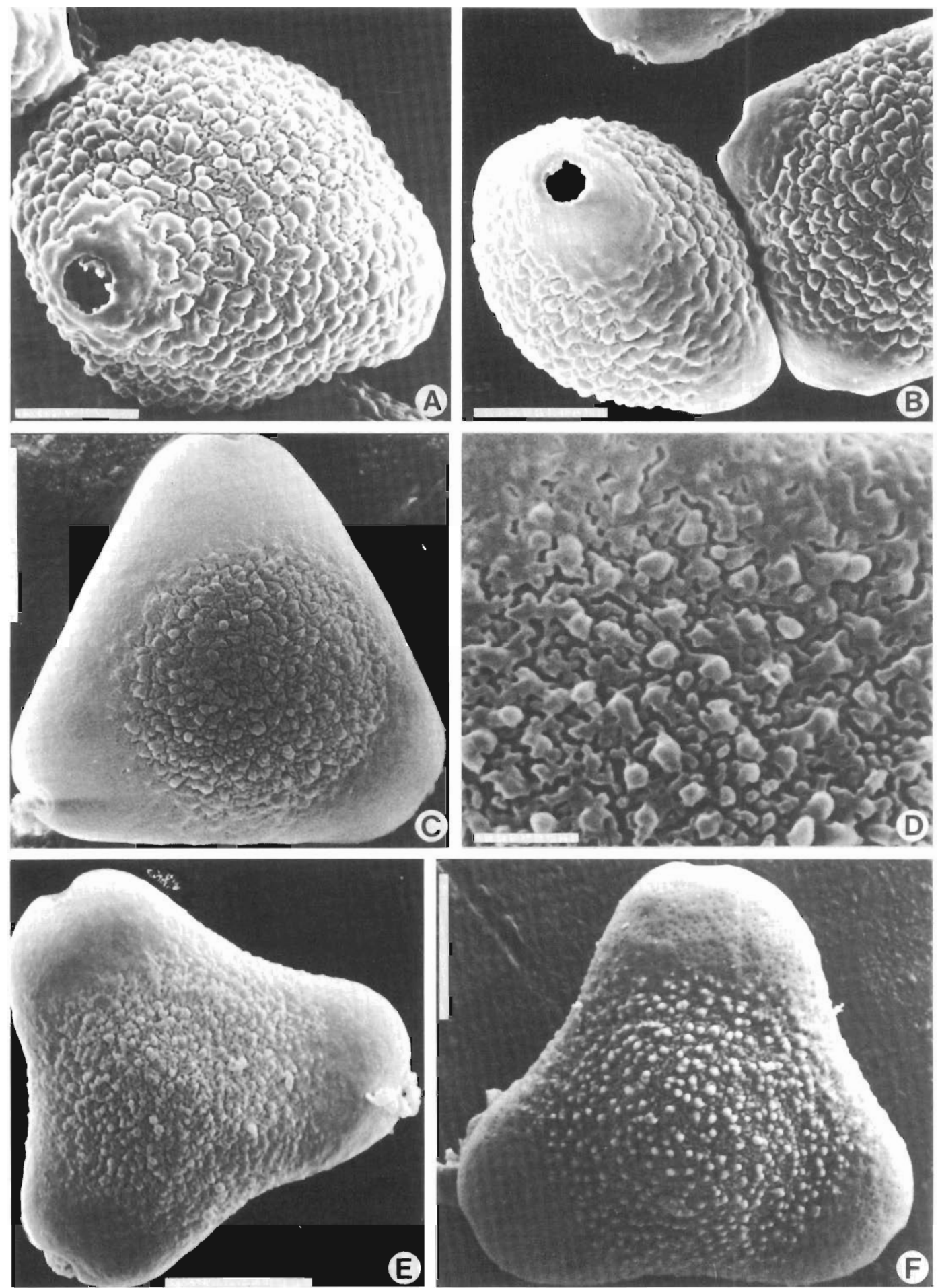

Fig. 4: Tipo VII: A, Helicteres vuarame (Krapovickasetal. 38006); B, H. Lhotzkyana var. Lhotzkyana (Schinini etal. 20321). Tipo VIII: C-D,H. brevispira (Rossi SPF 23075); E, H. macropetala(Krapovickaset al. 38056); F, H. rufipila (Ganev 1317). Escalas=10 $\mu \mathrm{m}(\mathrm{A}, \mathrm{B}, \mathrm{C}, \mathrm{E}, \mathrm{F}) ; 2 \mu \mathrm{m}(\mathrm{D})$. 

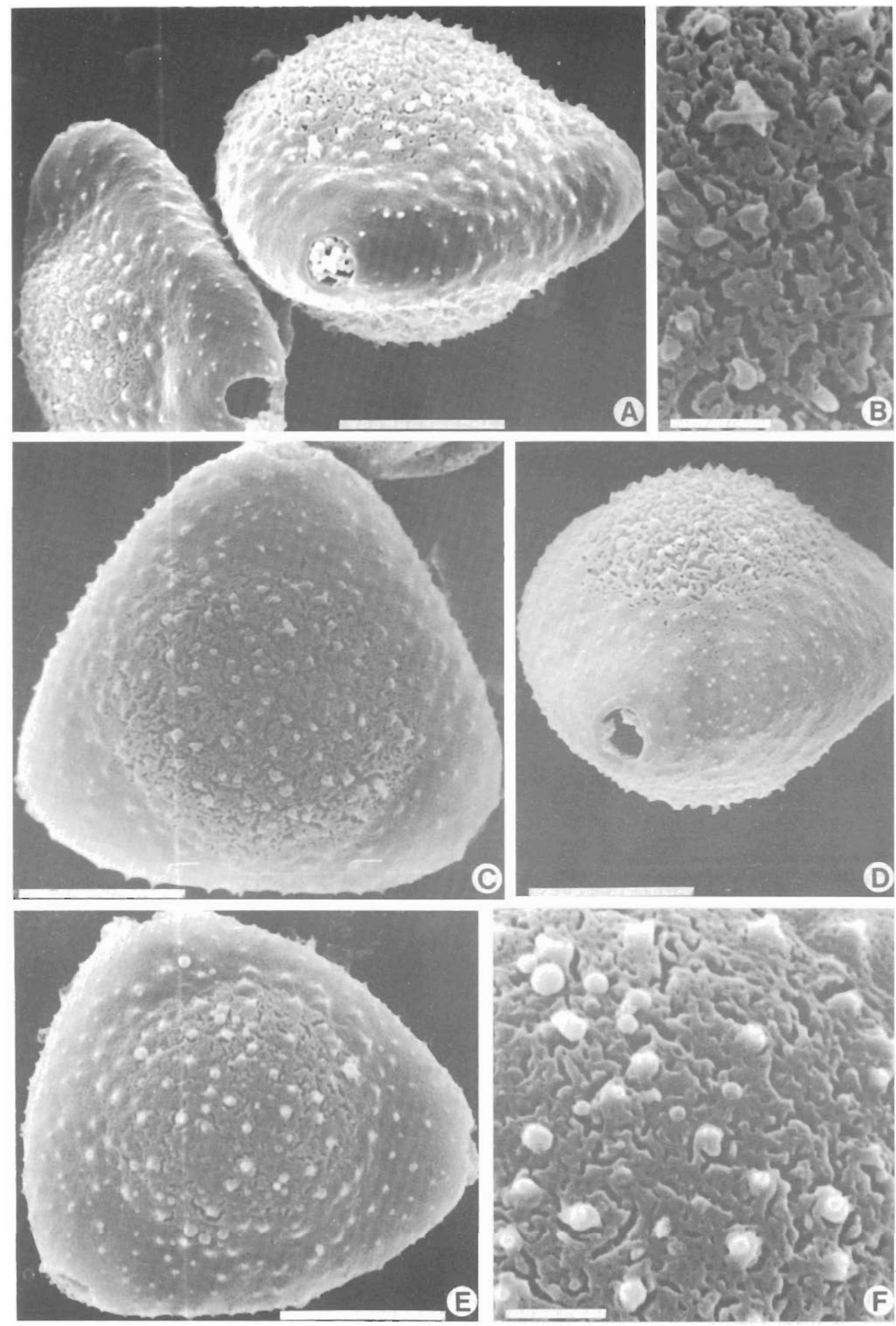

Fig. 5. Tipo IX: A-C, Helicteres sacarolha (Menezes SPF 22582); D, H muscosa (Krapovickas et al. 38784); E-F, H Eitenii (Krapovickas et al. 37840). Escalas $=10 \mu \mathrm{m}(\mathrm{A}, \mathrm{C}, \mathrm{D}, \mathrm{E}) ; 2 \mu \mathrm{m}(\mathrm{B}, \mathrm{F})$. 

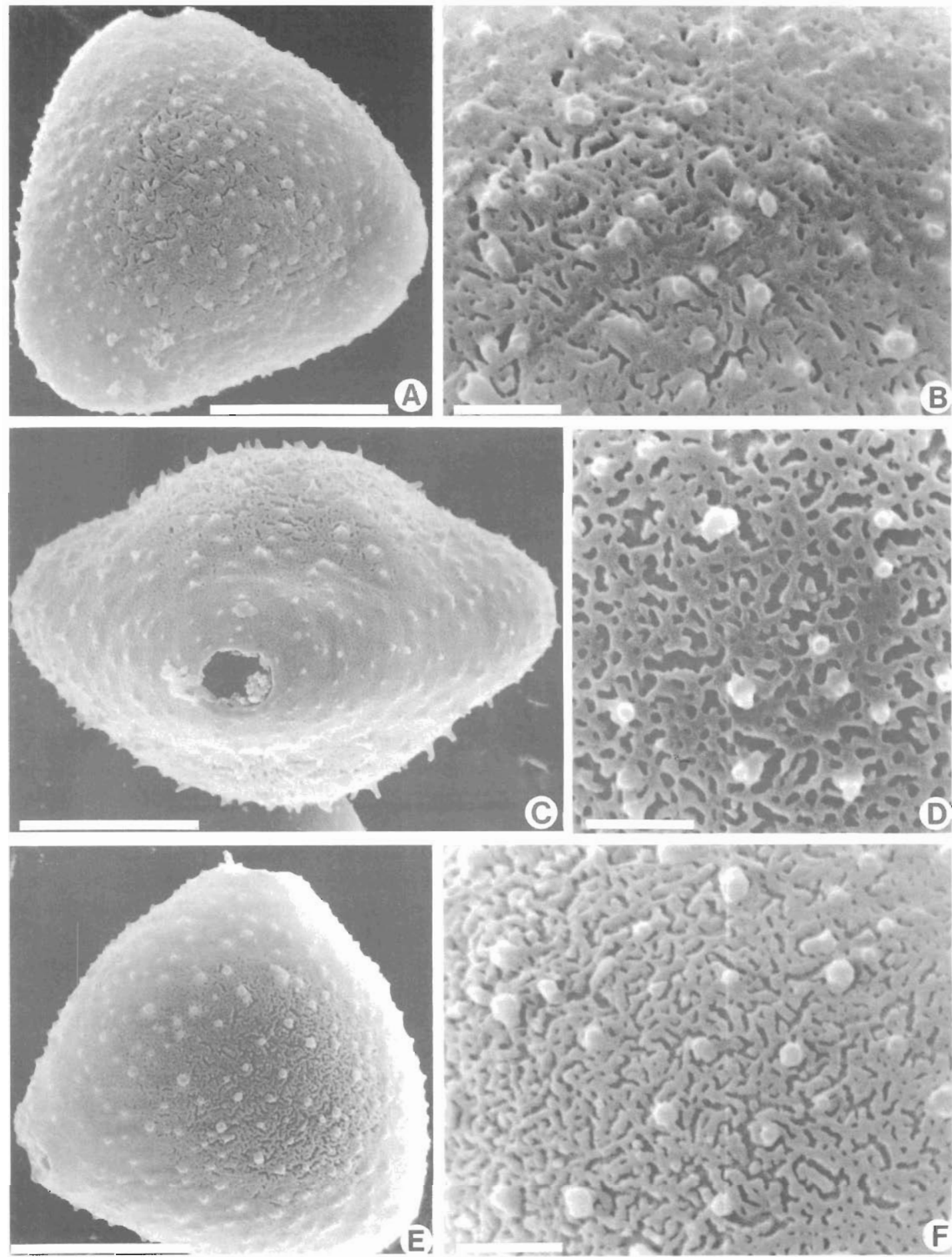

Fig. 6. Tipo IX: A-B, Helicteres Andersonii (Hatschbach et al. 60225); C-D, H. ovata (Sucre 3361); E-F, H. velutuna (Carvalho et al. 1768). Escalas $=10 \mu \mathrm{m}(\mathrm{A}, \mathrm{C}, \mathrm{E}) ; 2 \mu \mathrm{m}(\mathrm{B}, \mathrm{D}, \mathrm{F})$. 


\section{Clave para diferenciar los tipos polínicos de Helicteres}

1. Granos con verrugas supratectales bien diferenciadas o desde muy atenuadas a totalmente ausentes.

2. Verrugas bien diferenciadas.

3. Verrugas localizadas principalmente en los mesoporios

3. Verrugas distribuidas en todo el grano (excepto en anillos).

Tipo III

4. Téctum imperforado o con escasas perforaciones, escábrido

4. Téctum perforado o microrreticulado.

Tipo V

5. Superficie microrreticulada

5. Superficie tectado-perforada.

6. Perforaciones normalmente circulares, verrugas menores de $1 \mu \mathrm{m}$

6. Perforaciones usualmente alargadas, verrugas de $1 \mu \mathrm{m}$ o más de diámetro, a veces fusionadas entre sí

2. Verrugas muy atenuadas o totalmente ausentes (superficie psilada)

Tipo VII

Tipo II

1. Granos con báculas o microespinas supratectales.

7. Téctum con perforaciones y báculas distribuidas en toda la superficie del grano

7. Téctum con perforaciones localizadas predominantemente en las zonas polares.

8. Microespinas concentradas en las zonas polares

8. Microespinas distribuidas en toda la superficie del grano

Tipo VIII

Tipo IX

Relación entre el polen de Helicteres y otros géneros de la tribu Helictereae

Con fines comparativos y para establecer el grado de afinidad palinológica entre Helicteres y géneros vecinos, se analizó la morfología polínica de algunas especies pertenecientes a géneros ubicados clásicamente en la tribu Helictereae.

Neoregnellia cubensis Urb. (Fig. 7, A-B):

Granos 3-porados; isopolares y radiosimétricos, oblatos. Ámbito triangular, angulaperturado; mesoporios rectos a suavemente convexos. Tamaño de los granos mediano, $\mathrm{P}=18,5$ $(21,5) 26 \mu \mathrm{m}, E=26,6(28,5) 31,5 \mu \mathrm{m}$. Poros circulares a levemente alargados en sentido meridional, de 2-4 um de diámetro. Exina delgada, $1 \mu \mathrm{m}$ de espesor, siendo la sexina aproximadamente del mismo espesor que la nexina y engrosada alrededor de la aperturas $(1,4 \mu \mathrm{m})$. Sexina columelada, tectada a tectado-perfora- da, microequinada. Con el MEB se observa una clara diferenciación entre las áreas polares y la zona ecuatorial. En los polos, el téctum es perforado-fosulado; perforaciones del orden de 0,1 $\mu \mathrm{m}$ y fósulas de, aproximadamente, $1 \mu \mathrm{m}$ de largo. En los mesoporios, el téctum es casi continuo, microfoveolado, con algunas perforaciones aisladas. Las espínulas, de aproximadamente $1 \mu \mathrm{m}$ de alto en los polos, se atenúan hasta casi desaparecer en mesoporios y aperturas.

Género monoespecífico, propio de Cuba y encontrado también en la República Dominicana.

Material estudiado: REP. DOMINICANA. Bánica. Cueva de San Francisco. 18.IX.1977, Cicero et al. 8415 (CTES), PAL-CTES 5591.

\section{Kleinhovia hospita L.}

(Fig. 7, C-D):

Granos 3-porados; isopolares y radiosimétricos, suboblatos. Ámbito triangular, angulaperturado; mesoporios levemente convexos. 
Tamaño pequeño, $\mathrm{P}=14-16 \mu \mathrm{m}, \mathrm{E}=16,8-20 \mu \mathrm{m}$. Poros pequeños, de 1,5 $\mu \mathrm{m}$ de diámetro, poco visibles con el MO. Exina delgada y de espesor uniforme en todo el grano $(1 \mu \mathrm{m})$, sexina más gruesa que la nexina, columelada y microrreticulada. Microrretículo con lúmenes muy irregulares, de aproximadamente $1 \mathrm{\mu m}$ de largo, con muros sinuosos de 0,2 $\mu \mathrm{m}$ de ancho; hacia el ecuador se reduce el tamaño de los lúmenes.

Género monoespecífico, distribuido en trópicos y subtrópicos del Viejo Mundo.

Material estudiado: PUERTO RICO. Mayagüez, Cult. Campus Univ. Puerto Rico, 11.XI.1993, Nee 44184 (CTES), PAL-CTES 5596.

\section{Reevesia thyrsoidea Lindl.}

(Fig. 8, A-B):

Granos 3-brevicolporados; isopolares, radiosimétricos y suboblatos. Ámbito subtriangular angulaperturado; mesocolpios convexos. Tamaño de los granos pequeño, $\mathrm{P}=10,3-14,7 \mu \mathrm{m}$, $\mathrm{E}=15,5-17,5 \mu \mathrm{m}$. Colpos cortos y angostos, de 2,8-3,5 $\mu \mathrm{m}$ de largo x 1-1,4 $\mu \mathrm{m}$ de ancho; endoaperturas circulares a levemente lolongadas, de 2,8-3,5 $\mu \mathrm{m}$; en sentido meridional ambas aperturas (externa e interna) tienen el mismo diámetro no así en sentido ecuatorial, de modo que sólo es posible observar las endoaperturas con MO. Exina de 1,2 $\mu \mathrm{m}$ de espesor, sexina $(0,7 \mu \mathrm{m})$ más gruesa que la nexina $(0,5 \mu \mathrm{m})$; alrededor de las endoaperturas la nexina se engrosa hasta $1 \mu \mathrm{m}$. Sexina tectado-perforada, supra-reticulada. El supraretículo es angustimurado, con muros levemente sinuosos, de $0,3 \mu \mathrm{m}$ de ancho y lúmenes irregulares, de 0,4-1,5 $\mu \mathrm{m}$ de ancho x 0,6-2,5 $\mu \mathrm{m}$ de largo en los polos, el tamaño de los lúmenes se reduce hacia la zona ecuatorial.

Género con ca. de 3 especies distribuidas en el SE de Asia.

Material estudiado: Annam, Quang-tri, 2.VI.1924, Poilaine 10720 (P), PAL-CTES 5815.

\section{Veeresia clarkii Monach. et Moldenke} (Fig. 8, C-D):

Granos 4-zonobrevicolporados; isopolares, radiosimétricos y suboblatos a oblatos. Ámbito cuadrangular planaperturado o angulaperturado o a veces subcircular-periaperturado; mesocolpios rectos a convexos. Tamaño de los granos pequeño, $\mathrm{P}=13,3(14,8) 17,7 \mu \mathrm{m}, \mathrm{E}=15,5$ $(18,7) 24,5 \mu \mathrm{m}$. Colpos cortos y angostos, de 3-4 $\mu \mathrm{m}$ de largo $\times 1-1,4 \mu \mathrm{m}$ de ancho; endoaperturas circulares a lolongadas, de 1,4-3,8 $\mu \mathrm{m} \times 1,4-2$ $\mu \mathrm{m}$. Exina de 1-1,2 um de espesor, siendo la sexina aproximadamente del mismo espesor que la nexina, ésta se engrosa levemente alrededor de las endoaperturas. Sexina tectado-perforada, supra-reticulada. El supra-retículo es angustimurado, con muros levemente sinuosos, de $0,3 \mu \mathrm{m}$ de ancho y lúmenes irregulares, de 0,4-1,5 um de ancho x 0,6-2,5 $\mu \mathrm{m}$ de largo en los polos, más pequeños hacia el ecuador. Las perforaciones se localizan principalmente en la base de los muros.

Género hasta la fecha constituido por una sóla especie nativa de México. De acuerdo a estudios de S. Solheim (WIS) aun no formalmente publicados, Veeresia debería asimilarse al género asiático Reevesia Lindl. que contaría con una segunda especie mexicana.

Material estudiado: MÉXICO, Hidalgo, Mun. Chapuhuacán, $14 \mathrm{~km}$ SW of Tamazunchale, 7.VII.1984, Solheim 1765 (CTES), PAL-CTES 5588.

\section{Ungeria floribunda Schott et Endl. (Fig. 8, E-F):}

Granos 5-zonobrevicolpados, isopolares y radiosimétricos. Oblatos, de ámbito circular Tamaño de los granos pequeño, $\mathrm{P}=12,6$ (14) 16 $\mu \mathrm{m}, \mathrm{E}=16$ (18) $22 \mu \mathrm{m}$. Colpos cortos y angostos, de $4-5 \mu \mathrm{m}$ de largo x 1-1,5 $\mu \mathrm{m}$ de ancho. Exina de $1 \mu \mathrm{m}$ de espesor, siendo la sexina más gruesa $(0,7 \mu \mathrm{m})$ que la nexina $(0,3 \mu \mathrm{m})$; en el margen de los colpos la nexina forma costillas de 1-1,5 $\mu \mathrm{m}$ de espesor. Sexina columelada, reticulada en apocolpios y psilada en mesocolpios. Retículo heterobrocado, con lúmenes de 0,5-1 $\mu \mathrm{m}$ de ancho x 1-2 $\mu \mathrm{m}$ de largo, entre los que se intercalan otros más pequeños, del orden de $0,3 \mu \mathrm{m}$ de diámetro; muros, más o menos rectilíneos, de aproximadamente 0,2 $\mathrm{mm}$ de ancho. Hacia los mesocolpios el tamaño de los lúmenes se reduce gradualmente hasta constituir un téctum continuo, suavemente foveolado. 

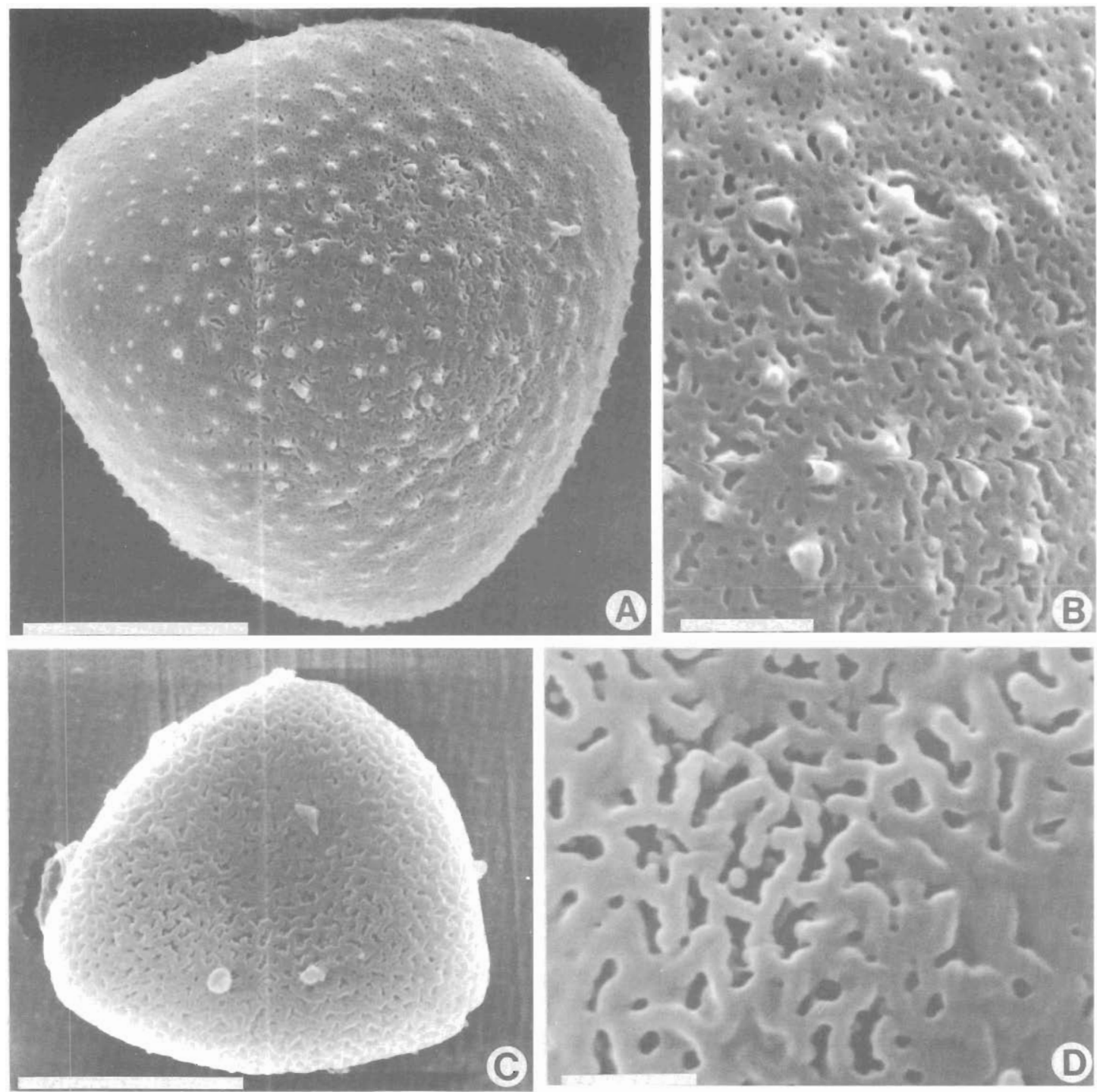

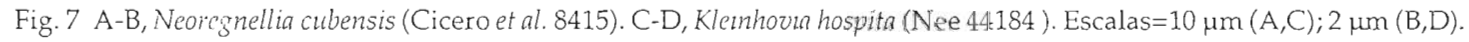



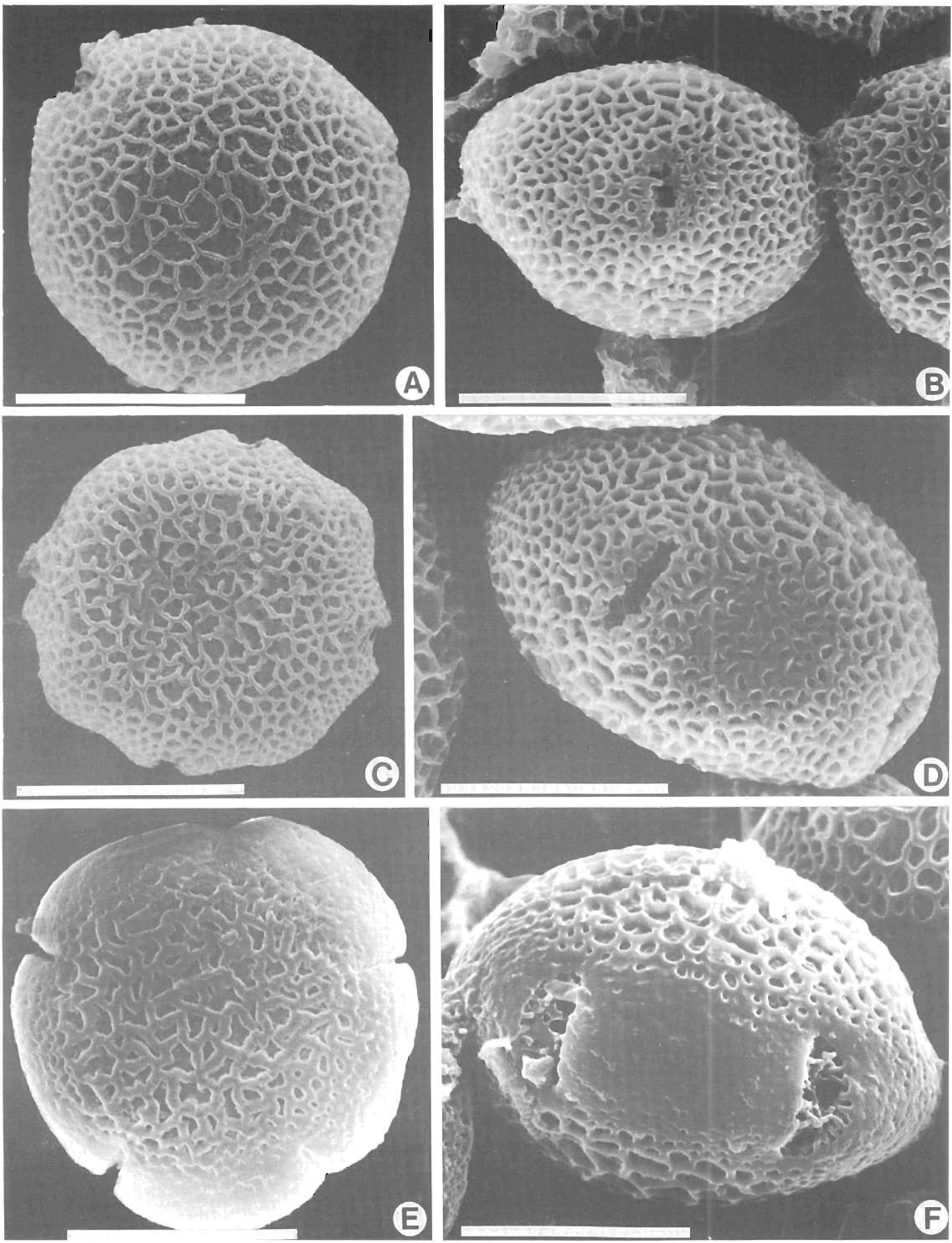

Fig. 8. A-B, Reevesia thyrsoidea (Poilaine 10720). C-D, Veeresta clarkil (Solheim 1765). E-F, Ungerta floribunda (Ralston 13). Escala $=10 \mu \mathrm{m}$. 

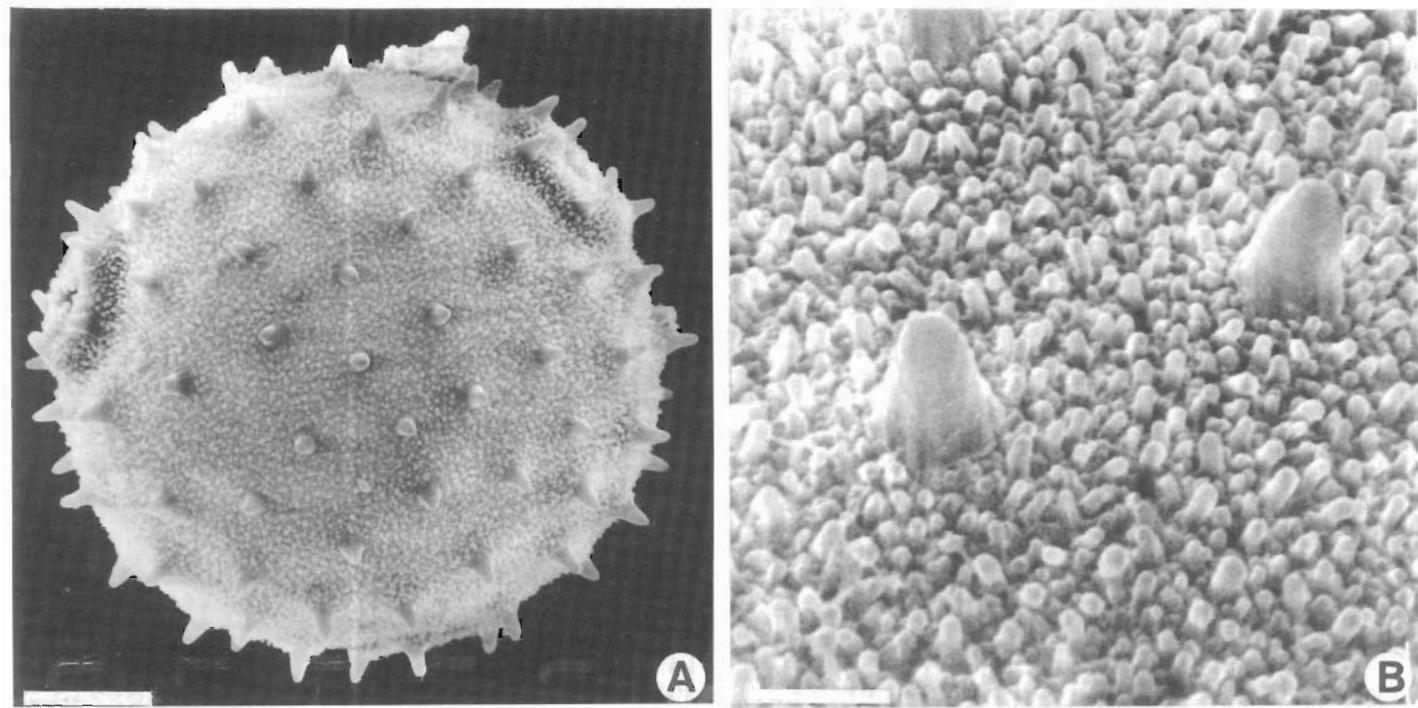

Fig. 9. A-B, Pterospermun acerifolium (Daniel s/n). Escalas $=10 \mu \mathrm{m}(\mathrm{A}) ; 2 \mu \mathrm{m}(\mathrm{B})$. lia.

Género monoespecífico propio de Austra-

Material estudiado: AUSTRALIA. Norfolk Island, Mt. Bates, XII.1963, Ralston 13 (A), PAL-CTES 5706.

\section{Pterospermum acerifolium Willd.}

(Fig. 9, A-B):

Granos 3-porados, isopolares, radiosimétricos y esferoidales. Tamaño mediano a grande, entre 45-55 $\mu \mathrm{m}$ de diámetro. Exina de 1,5-2 $\mu \mathrm{m}$ de espesor, siendo la sexina $(0,5-0,7 \mu \mathrm{m})$ más delgada que la nexina $(1-1,5 \mu \mathrm{m})$, alrededor de los poros la nexina se engrosa notablemente $(3,5-4 \mu \mathrm{m})$. Sexina tectada, columelada, con espinas supratectales. Con el MEB se observa que el téctum es perforado y la superficie densamente micro-granulada, baculada; las espinas, obtusas y de hasta $5 \mu \mathrm{m}$ de largo, se distribuyen uniformemente en todo el grano.

Género de alrededor de 18 especies, propio del E de Asia.

Material estudiado: BRASIL. São Paulo, Campinas, Cult. Faz. Santa Elisa, 25.X.1969, Daneel s/n (CTES), PAL-CTES 5597.

\section{Clave de géneros de la tribu Helictereae}

1. Granos mayores de $40 \mu \mathrm{m}$ de diámetro, esferoidales y equinados

1. Granos menores de $40 \mu \mathrm{m}$, oblatos a suboblatos.

Pterospermum

2. Granos 3 a 5 zono-brevicolp(or)ados, tectado-perforados suprarreticulados, con lúmenes mayores de $1 \mu \mathrm{m}$ en los polos.

3. Granos 5 colpados, retículo sólo en apocolpios, mesocolpios psilados a levemente fosulados

3. Granos 3 ó 4 colporados, retículo en toda la superficie.

Ungeria

4. Granos 3 brevicolporados

4. Granos 4 brevicolporados

Veeresia

Reevesia 
2. Granos 3 zono-porados.

5. Exina micro-reticulada, lúmenes de aproximadamente $1 \mu \mathrm{m}$ en los polos y más pequeños en mesoporios

5. Exina con ornamentación variada, cuando micro-reticulada, lúmenes del orden d diámetro y con verrugas supratectales.

6. Exina micro-equinada en todo el grano y con perforaciones y fósulas limitadas a los polos

Neoregnellia y Helicteres (secc. Sacarolha y Orthothecium, tipo IX).

6. Exina generalmente verrugada y con perforaciones y / o fósulas en todo el grano; a veces microequinada, pero en este caso tanto microespinas como perforaciones se concentran en los polos; raro con báculas supratectales o con téctum microrreticulado-supraverrugado

Helicteres

\section{Discusión y conclusiones}

La comparación de la morfología del polen de Helicteres con la de los géneros restantes de la tribu Helictereae: Neoregnellia, Kleinhovia, Reevesia, Veeresia, Ungeria y Pterospermum pone de manifiesto que los géneros más afines son Neoregnellia y Kleinhovia. La afinidad más estrecha se establece con Neoregnellia, como se ve en la clave de géneros, que posee el tipo polínico IX de Helicteres, presente en la secc. Sacarolha y en algunas especies de la secc. Orthothecium (Fig. 7, A-B). Mientras que Kleinhovia se diferencia de Helicteres sólo por la ornamentación de la exina, que es microrreticulada y sin elementos supratectales (Fig. 7, C-D); la ausencia de elementos esculturales sobre el téctum relaciona este género con el Tipo II, en el cual la exina es psilada.

Respecto a Reevesia, Veeresia y Ungeria, el grado de afinidad con Helicteres es menor ya que en estos géneros los granos son brevicolp(or)ados, con téctum supra-reticulado. Estos tres géneros, sin embargo están muy relacionados entre sí por la morfología del polen. Se presentan diferencias vinculadas con las aperturas y con la ornamentación de la exina en la zona ecuatorial. En Reevesia thyrsoidea y Veeresia clarkii los granos son colporados y reticulados en toda la superficie y únicamente se diferencian entre sí por el número de aperturas, 3 y 4 respectivamente. En Ungeria floribunda los granos son 5-colpados y reticulados sólo en los apocolpios, mientras que la zona ecuatorial es tectada psilada levemente foveolada (Fig. 8).

Pterospermum, con granos grandes, esferoidales y equinados, es el que más se apar- ta de Helicteres, presentando más afinidad con géneros de la tribu Dombeyeae, como ya lo había sugerido Rao (1950), y confirmando su separación de la tribu Helictereae (Fig. 9).

En cuanto a Helicteres, si bien el polen es bastante uniforme en lo que se refiere a la forma y tamaño de los granos y al tipo y número de las aperturas, presenta diferencias en la escultura de la exina que revisten importancia taxonómica. De acuerdo con la escultura se pueden reconocer 9 tipos polínicos, que se correlacionan en parte con las secciones reconocidas por Cristóbal (2001), y que a su vez, muestran entre sí una cierta variación gradual. En los diagramas A y B se han representado dos posibles sentidos evolutivos de la exina; en ellos las secciones están marcadas por medio de rectángulos cuyos tamaños guardan relación con el número de especies, y la dirección de la evolución está indicada por las flechas. Las secciones asiáticas Helicteres y Orthocarpaea se ubican en la parte inferior de los diagramas por poseer los tipos polínicos más simples dentro del género. En ambas los granos de polen poseen téctum uniformemente perforado; Helicteres, constituida sólo por $H$. isora, se caracteriza por poseer báculas supratectales (Tipo I), este carácter es único en el género; Orthocarpaea con 21 especies presenta cierta variación; de esta sección se analizaron sólo 6 especies, en cuatro de ellas el téctum es psilado o con verrugas apenas insinuadas (Tipo II), mientras que en las dos especies restantes, H. hirsuta y $H$. viscida, es netamente verrugado (Tipo VI). Sin embargo, el paso de un tipo polínico a otro es gradual, o sea se presenta una serie más o menos continua entre granos psilados y granos supra-verrugados. 
Tipos polínicos
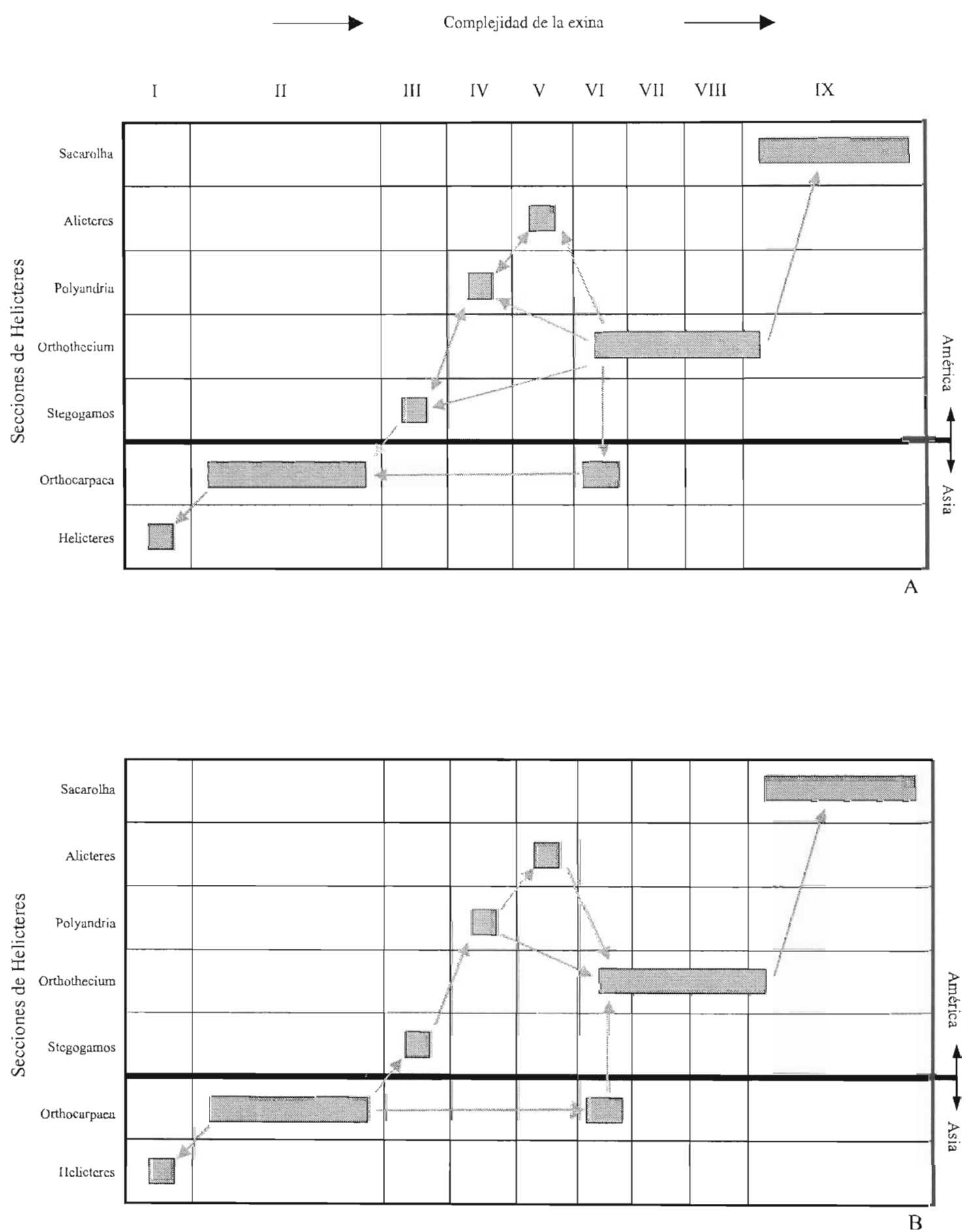

Diagramas de los posibles sentidos evolutivos de la exina de Helicteres En A, a partir del Tipo VI y en B, a partir del Tipo II 
Cabe la posibilidad que el estudio de todas las especies de esta sección muestre una mayor variación.

En estrecha relación con las secciones asiáticas se encuentran, por un lado, la secc. Stegogamos y, por otro, la secc. Orthothecium. En Stegogamos, con una sóla especie H. Vegae endémica del SW de México, los granos son en parte psilados y en parte verrugados (Tipo III); esta especie es considerada por Cristóbal (1987) como nexo entre los dos continentes por ser la única en América en presentar una combinación de caracteres que son propios de la secc. Orthocarpaea, criterio que es apoyado por el polen.

Vinculadas a Stegogamos se encuentran las otras dos secciones monoespecíficas estrictamente caribeñas, Polyandria y Alicteres, en las que los granos de polen poseen características peculiares a nivel del téctum, que es microrreticulado en la primera (Tipo IV) y escabrado escasamente perforado en la segunda (Tipo V), con verrugas supratectales distribuidas en toda la superficie; este carácter las relaciona con la secc. Orthothecium.

Orthothecium es la sección americana de mayor difusión y es, además, la que presenta la más amplia gama de variación, tanto en lo que respecta a la morfología de los granos de polen como al resto de los caracteres exomorfológicos. En esta sección se pueden reconocer cuatro tipos polínicos: VI, VII, VIII y IX. Los Tipos VII y VIII son exclusivos de esta sección. En el primero los granos son uniformemente perforado-fosulados y con verrugas de mayor tamaño, parcialmente fusionadas en los polos o, como en $H$. Lhotzkyana, casi totalmente fusionadas en los mesoporios. Esta especie constituiría el nexo con el Tipo VIII, en el que las perforaciones y fósulas se concentran en los polos mientras que las zonas ecuatoriales (mesoporios) son tectado-psiladas o levemente escabradas; en los polos se concentran también los elementos supratectales, que pueden ser más o menos redondeados (microverrugas) o puntiagudos (microespinas). Los Tipos VI y IX son comunes con otras secciones. Las especies caribeñas $H$. jamaicensis, $H$. semitriloba y $H$. trapezifolia y la brasileña $H$. laciniosa (secc. Orthothecium) comparten el mismo tipo de polen con las especies asiáticas $H$. hirsuta y H. viscida (secc. Orthocarpaea), granos uniformemente perforados y verrugados (Tipo VI). Es interesante señalar que estas dos especies constituyen una excepción dentro de la secc. Orthocarpaea, por presentar inflorescencias plurifloras como algunas especies de la secc. Orthothecium. Otro grupo de especies constituido por $H$. Andersonii, $H$. biflexa, $H$. longepedunculata, $H$. ovata y $H$. velutina, posee el mismo tipo de polen que se presenta en la secc. Sacarolha (Tipo IX).

Sacarolha, se ubica en el extremo superior derecho de los diagramas por presentar un tipo polínico considerado como "avanzado" dentro de Helicteres, los granos muestran una clara diferenciación entre las áreas polares y la zona ecuatorial: en los polos se concentran las perforaciones y fósulas, que pueden llegar a fusionarse totalmente, y las microespinas son especialmente notables, mientras que hacia el ecuador el téctum es continuo o apenas foveolado y las microespinas están muy reducidas en tamaño (Tipo IX). Todas las especies estudiadas de esta sección presentan el mismo tipo de polen con ligeras variaciones en el tamaño y fusión de las perforaciones. Esta uniformidad se manifiesta también en otros caracteres exomorfológicos, Sacarolha es una de las secciones mejor definidas dentro de Helicteres. Un carácter que caracteriza a esta sección es la ausencia de nectarios extraflorales que sí están presentes en el resto de las especies del género, excepto en un grupo de especies de Orthothecium, algunas de las cuales (H. Andersonii, H. biflexa, H. longepedunculata) tienen, además en común con Sacarolha el tipo de polen.

Los dos tipos polínicos ubicados en los extremos poseen una morfología bien diferenciada por sus elementos supratectales: en el Tipo I, báculas y en Tipo IX, microespinas. Entre un extremo y otro, se encuentran los "tipos verrugados" o sea los tipos polínicos que presentan como elemento supratectal, verrugas; éstas pueden presentarse desde apenas insinuadas hasta bien desarrolladas y desde distribuidas en toda la superficie hasta concentradas en los polos. Estos "tipos verrugados" muestran una amplia variación y las modificaciones que caracterizan cada tipo polínico (II, III, IV, V, VI, VII y VIII) están, en mayor o menor grado, correlacionadas entre sí. 
En la interpretación del sentido evolutivo de la exina cabrían dos posibilidades. La primera posibilidad, que se muestra en el diagrama A, sería que a partir del Tipo VI, o sea granos uniformemente perforados y verrugados, la exina habría evolucionado en distintas direcciones. Por un lado, hacia una mayor complejidad que termina con el Tipo VIII, en el que las verrugas (microverrugas) se concentran en los polos mezcladas con elementos más o menos puntiagudos (microespinas). A partir de este tipo se habría diferenciado el Tipo IX. Por otro lado, se habría producido una diversificación en distintas direcciones que daría lugar a modificaciones que conducirían gradualmente a una simplificación de la exina y que culminaría en la ausencia de elementos supratectales, Tipo II. A partir de este tipo se habría diferenciado el Tipo I.

La segunda posibilidad, representada en el diagrama $B$, es que el sentido evolutivo de la exina haya sido unidireccional. En este caso a partir del Tipo II la exina habría adquirido cada vez una mayor complejidad dando lugar a tipos polínicos que están conectados entre sí por formas intermedias o de transición entre unos y otros.

Se puede concluir que la morfología del polen aporta elementos importantes a la taxonomía de Helicteres, apoyando los cambios propuestos por Cristóbal (2001) en la estructura infragenérica del género. Así mismo, proporciona información para interpretar el posible origen del mismo.

Las secciones monoespecíficas poseen tipos polínicos característicos o sea pueden definirse también por el polen. Las secciones con mayor número de especies están vinculadas, en cier- ta medida, entre sí y estas conexiones tanto polínicas como exomorfológicas pondrían de manifiesto la coherencia del género.

\section{Bibliografía}

CHAUDHURI, S. K., 1969. Contribution to the pollen morphology of Sterculiaceae. J. Sen. Mem. Com. and Bot. Soc. of Bengal, Calcuta, India: 229-238.

CRISTÓBAL, C. L., 1987. Un Helicteres nuevo de México, nexo entre las especies asiáticas y americanas. Bonplandia 6(1): 71-81. Figs. 1-2.

—. 2001. Taxonomía del género Helicteres (Sterculiaceae). Revisión de las especies americanas. Bonplandia 11(1-4): 1-206.

ERDTMAN, G., 1966. Pollen Morphology and Plant Taxonomy. Angiosperms. Hafner Publ. Co., New York.

HUANG, T.- Ch., 1967. Pollen grains of Formosa plants. Taiwania 13: 15-110.

- 1972. Pollen Flora of Taiwan. National Taiwan University, Botany Department Press. Taiwan.

MELHEM, T., M.S.F. SILVESTRE \& N.M.C. LUCAS, 1976. Pollen morphological studies inSterculiacene. Hoehnea 6: 23-32.

PETROV, SI. \& Ts. DRAZHEVA-STAMATOVA, 1972. Reevesia Lindl. fossil pollen in the tertiary sediments of Europe and Asia. Pollen et Spores 14(1): 79-95.

PUNT, W., S. BLACKMORE, S. NILSSON \& A. LE THOMAS, 1994. Glossary of Pollen and Spore Terminology. LPP Foundation, LPP Contributions Serie $N^{\circ} 1$. University of Utrecht, The Netherlands.

RAO, C. V., 1950. Pollen grains of Sterculiaceae.J. Indian Bot. Soc. 29(2): 130-137.

SALGADO-LABOURIAU,M.L.S., 1973.Stercultaceae.p. 165167. Contribuição à Palinológia dos Cerrados. Academia Brasileira de Ciências.

WINKLER, H., 1906. Beiträge zur Morphologie und Biologie tropischer Blüten und Früchte. Bot. Jahrb. Syst. Achtunddreissigster Band: 254-258

ZEBE, V., 1915. Monographie der Sterculiaceen-Gattungen Kleinhovia, Helicteres, Reevesia, Ungeria und Pterospermum. Inaugural-Dissertation. Kgl. Botanischen Garten zu Breslau: 1-63 